\title{
Klimaschutzverfassungsrecht: Klima-Staatszielbestimmungen im Föderalismus
}

\author{
Ines Härtel*
}

(c) Der/die Autor(en) 2020

Klimaschutz wird zunehmend Aufgabe des Verfassungsrechts in föderaler Gestaltung. Kontrovers ist dabei die Aufnahme einer Klima-Staatszielbestimmung. Welche Wirkungen ergeben sich für Grundrechte, welcher Mehrwert entsteht gegenüber der aktuellen Rechtslage? Welche Problematik birgt der „Klimavorrang" - gerade in Bezug auf Schutzgüterkonflikte? Hier ist auch der Blick auf die Entwicklung des Verfassungsrechts anderer Staaten aufschlussreich.

\section{Einführung}

Für einen effektiven globalen Klimaschutz kommt es nicht nur auf die konkreten Maßnahmen zum richtigen Zeitpunkt $t^{1}$ an, sondern ebenso auf die entsprechenden rechtlichen Grundlagen. Diese sind allerdings hoch komplex, mehrdimensional strukturiert und in Mehrebenenystemen eingebettet. Dies betrifft das Klimavölkerrecht mit der Klimarahmenkonvention und dem Übereinkommen von $\mathrm{Pa}$ ris $^{2}$ sowie die Weiterentwicklung des EU-Klimarechts im Sinne des „Green Deals“3 oder staatliche Klimagesetze, wie z. B. in Deutschland das Klimaschutzgesetz des Bundes ${ }^{4}$ so-

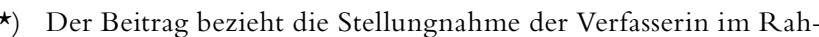
men der Anhörung im Niedersächsischen Landtag am 20.1.2020 zur Änderung der Landesverfassung im Hinblick auf die Aufnahme einer expliziten Klima-Staatszielbestimmung ein.

1) $\mathrm{Zu}$ erforderlichen Maßnahmen, insbesondere dem noch verbleibenden globalen Treibhausgasbudget, s. IPCC-Sonderbericht zu $1,5^{\circ} \mathrm{C}$ globale Erwärmung, Zusammenfassung für Entscheidungsträger, 2018, deutschsprachige Übersetzung, Stand 20.7.2020, abrufbar unter https://www.ipcc.ch/site/assets/uploads/2019/03/SR1.5-SPM_de_barrierefrei-2.pdf.

2) Vgl. Jahrmarkt, in: Härtel (Hrsg.), Forum Umwelt-, Agrar- und Klimaschutzrecht, Bd. 9, 2016, S. 61 ff., S. 261 ff., S. 344 ff. (auch zu Vorschlägen im Hinblick auf einen effektiven Weltklimavertrag); Kreuter-Kirchhof, DVBl. 2017, $97 \mathrm{ff}$.

3) Komm., Mitt. v. 11.12.2019, Der europäische Grüne Deal, $\operatorname{COM}(2019) 640$ final.

4) Gesetz zur Einführung eines Bundes-Klimaschutzgesetzes und zur Änderung weiterer Vorschriften v. 12.12.2019, BGBl. I, S. 2513.

5) Hierzu Flaskühler, Föderale Klimaschutzgesetzgebung in Deutschland im Lichte des wohlgeordneten Rechts, 2018, S. $132 \mathrm{ff}$., S. $340 \mathrm{ff}$; Schilderoth/Papke, Strukturelemente der Landesklimaschutzgesetze, Würzburger Berichte zum Umweltenergierecht Nr. 42 v. 27.5.2019.

6) Aktuell sind vier solche Verfassungsbeschwerden beim Bundesverfassungsgericht anhängig. Die erste wurde Ende November 2018 von einem Klägerbündnis bestehend aus dem Solarenergie-Förderverein Deutschland e. V. (SFV), dem Bund für Umwelt und Naturschutz Deutschland (BUND) und verschiedenen Einzelklägern

Prof. Dr. Ines Härtel,

Richterin am Bundesverfassungsgericht,

Inhaberin des Lehrstuhls für Öffentliches Recht, Verwaltungs-,

Europa-, Umwelt-, Agrar- und Ernährungswirtschaftsrecht,

Europa-Universität Viadrina,

Frankfurt (Oder), Deutschland wie Landesklimagesetze ${ }^{5}$. In besonderer Weise ist hier auch ein sich entwickelndes Klimaschutzverfassungsrecht gefordert, das die Dynamik und Komplexität der Bewältigung des Klimawandels integrativ aufnimmt. In diesem Kontext sind auch die beim Bundesverfassungsgericht eingelegten Klima-Verfassungsbeschwerden anzuführen. ${ }^{6}$

Im deutschen Föderalismus wird aktuell die explizite Verankerung des Klimaschutzes als Staatsziel in das Verfassungsrecht diskutiert. Als erstes Bundesland hat Hamburg im Februar 2020 den Klimaschutz als Staatsziel in die Verfassung aufgenommen. ${ }^{7}$ Die in Bayern und SchleswigHolstein versuchten Anläufe zur Verankerung des KlimaStaatsziels in die Verfassung sind bislang gescheitert. In Niedersachsen sind verschiedene Legislativvorschläge eingebracht und beraten worden. ${ }^{8}$

Auf Bundesebene gab es einen Gesetzentwurf der Fraktion Bündnis 90/Die Grünen ${ }^{9}$ zur Aufnahme des Klimaschutzes in das Grundgesetz. Art. 20a GG sollte danach um einen Satz ergänzt werden: „Für die Bundesrepublik Deutschland völkerrechtlich verbindliche Ziele und Verpflichtungen des Klimaschutzes binden alle staatliche Ge-

erhoben, zur Presseerklärung, Stand 20.7.2020, abrufbar unter https://www.bund.net/service/presse/pressemitteilungen/detail/news/verfassungsklage-Wegen-unzureichender-deutscherklimapolitik/ (der Schriftsatz, Stand 20.7.2020, abrufbar unter https://klimaklage.com/wp-content/uploads/2020/02/Klimaklage-Endfassung-Internet.pdf); Anfang 2020 folgten drei weitere Beschwerden mit Blick auf das Ende 2019 in Kraft getretene Bundes-Klimaschutzgesetz. In zwei davon sind die Beschwerdeführer in Deutschland wohnhafte Kinder und junge Erwachsene. Eine wird von Greenpeace und Germanwatch unterstützt (zu einer Zusammenfassung der Beschwerde, Stand 20.7.2020, abrufbar unter https://www.greenpeace.de/sites/www.greenpeace.de/ files/publications/klima-verfassungsbeschwerde.pdf); bei der anderen hat die Deutsche Umwelthilfe (DUH) mitgewirkt (Beschwerdeschrift, Stand 20.7.2020, abrufbar unter https://www. duh.de/fileadmin/user_upload/download/Pressemitteilungen/ Umweltpolitik/Klimaschutz/Verfassungsbeschwerde_Klimaklage_Linus_Steinmetz_et_al_final_geschw\%C3\%A4rzt-Anhang_01.pdf); im Rahmen einer vierten, ebenfalls von der DUH unterstützten, Verfassungsbeschwerde ziehen Menschen aus Bangladesch und Nepal vor das Bundesverfassungsgericht (Beschwerdeschrift, Stand 20.7.2020, abrufbar unter https://www.duh. $\mathrm{de} /$ fileadmin/user_upload/download/Pressemitteilungen/Umweltpolitik/Klimaschutz/Verfassungsbeschwerde_Klimaklage_ Yi_Yi_Prue_et_al_final_geschw\%C3\%A4rzt-Anhang_01.pdf); siehe ebenfalls BVerfG, Beschl. v. 28.10.2019, 2 BvR 966/19, juris; zu Klimaschutzklagen im Allgemeinen siehe z. B. Umweltprogramm der Vereinten Nationen, Klimawandel vor Gericht - ein globaler Überblick, Mai 2017; Frenz, UPR 2020, 1 ff.; Groß, EurUP 2019, $353 \mathrm{ff}$;; Saurer, ZUR 2018, $679 \mathrm{ff}$

7) Zur Begründung, Drs. 21/19680

8) Vorschlag von SPD und CDU: LT-Drs. 18/4839, Vorschlag von Bündnis90/Die Grünen: LT-Drs.18/4499, Vorschlag der FDP LT-Drs. 18/4494; hierzu im Rahmen der Anhörung im Nds. Landtag am 20.1.2020 die Stellungnahmen Härtel/Fischer/Schrader/Smollich.

9) BT-Drs. 19/4522; siehe hierzu auch Cremer, ZUR 2019, $278 \mathrm{ff}$. 
walt unmittelbar." ${ }^{10}$ Der Deutsche Bundestag hat diesen Vorschlag beraten und in seiner Sitzung vom 28.6.2019 abgelehnt. Unabhängig davon bleibt dieses Thema auf der Bundesebene in der Diskussion. Auf unionaler Ebene existiert seit dem Lissabon-Vertrag (2007) mit Art. 191, 4. Spiegelstrich AEUV die explizite Zielbestimmung der Bekämpfung des Klimawandels.

In rechtswissenschaftlicher Perspektive erfolgte bereits eine ausführlichere Auseinandersetzung mit der Frage, ob und inwieweit auf Bundesebene eine Klima-Staatszielbestimmung sinnvoll sein könnte. ${ }^{11}$ Auch wurde der bereits zitierte Gesetzentwurf der Fraktion Bündnis 90/Die Grünen kritisch reflektiert, insbesondere in den hierzu eingereichten Stellungnahmen zur Anhörung vor dem Ausschuss für Inneres und Heimat des Deutschen Bundestages. ${ }^{12} \mathrm{Im}$ Hinblick auf eine Klima-Staatszielbestimmung im Landesverfassungsrecht sind zwar auch bereits vor über zehn Jahren grundlegende Ideen vorgestellt worden ${ }^{13}$. Das Ideenspektrum hat sich allerdings seit dieser Zeit weiterentwickelt, wie insbesondere die Klima-Staatszielbestimmung in der Hamburgischen Verfassung und die im Niedersächsischen Landtag in 2019 eingereichten Gesetzentwürfe zur Änderung der Niedersächsischen Verfassung belegen. Die in Niedersachsen vorgelegten drei Gesetzentwürfe - gemeinsamer Entwurf der SPD-Fraktion und CDU-Fraktion, Entwurf der FDP-Fraktion, Entwurf der Fraktion Bündnis 90/Die Grünen -, das Klima-Staatsziel in Hamburg, aber auch die gescheiterten Entwürfe in SchleswigHolstein und Bayern zeigen exemplarisch unterschiedliche Gestaltungsoptionen zu einer Klima-Staatszielbestimmung in einer Landesverfassung auf. Sie sollen deshalb zum Anlass genommen werden, verschiedene grundsätzliche und spezielle Fragen im Hinblick auf die mögliche Verankerung einer Klima-Staatszielbestimmung im Landesverfassungsrecht zu beleuchten: Welche verfassungsrechtliche und verfassungspolitische Bedeutung sowie rechtspraktische Wirkung entfaltet eine Klima-Staatszielbestimmung im Landesverfassungsrecht? Weist sie einen föderalen Mehrwert gegenüber der Umweltstaatszielbestimmung des Art. 20a GG zum Schutz der natürlichen Lebensgrundlagen auf, die nach anerkannter Auffassung als Schutzgut auch das Klima umfasst und auch für die Bundesländer gilt? Auf welche (bundes)verfassungsrechtliche Grenzen stößt möglicherweise der Landesgesetzgeber bei der Ausgestaltung einer Klima-Staatszielbestimmung? Zur Beantwortung dieser Fragen sollen zuerst die dogmatischen Inhalte des Art. 20a GG dargelegt und sodann die Klimastaatszielbestimmung in Hamburg sowie die bisher vorgelegten Entwürfe zur Anderung der Landesverfassungen im Hinblick auf ein Klimastaatsziel beleuchtet werden. Des Weiteren gilt es, einen Blick auf Entwicklungen im ausländischen Klima-Verfassungsrecht zu werfen.

\section{Art. 20a GG als gesamtstaatliche Klima-Staatszielbestimmung}

\subsection{Historie}

Die deutsche Verfassungsgeschichte blickt mit Art. 150 Weimarer Reichsverfassung (1919) ${ }^{14}$ auf eine umweltbezogene Verfassungsnorm zurück, die aufgrund ihres eher programmatischen Charakters für den Staat damals bereits als Anregung (ohne zwingende Rechtsverbindlichkeit) diente. ${ }^{15}$ Die explizite Verankerung des Umweltschutzes in das Grundgesetz wurde als Idee und in ihrer möglichen Ausgestaltung seit den 1970'iger Jahren neu diskutiert. So gab es den Vorschlag für ein Grundrecht auf Umweltschutz. ${ }^{16}$ Die Sachverständigenkommission zu Staatszielbestimmungen/Gesetzgebungsaufträge plädierte in ihrem Bericht 1983 aber für die Etablierung eines Umwelt-Staatsziels. ${ }^{17}$ In diesem Bericht wurde auch erstmals der Begriff der Staatszielbestimmung definiert, der auf Hans Peter Ip- sen (1949) ${ }^{18}$ zurückgeht. Danach gehört es zur Rechtsnatur von Staatszielbestimmungen, dass sie verfassungsrechtlich bindende Wirkung entfalten. ${ }^{19}$ Der Durchbruch des Staatsziels Umweltschutz gelang im Kontext der Wiedervereinigung. Die aufgrund des Art. 5 Einigungsvertrag eingesetzte Gemeinsame Verfassungskommission erreichte nach Kontroversen im Jahre 1993 den Kompromiss der Formulierung des Art. 20a GG, der dann durch Gesetz vom 27. 10. 1994 in das Grundgesetz aufgenommen wurde. ${ }^{20}$

\subsection{Rechtsnatur und Rechtswirkungen}

Im Hinblick auf den Schutz des (globalen) Klimas ist mittlerweile in der Rechtsprechung des Bundesverfassungsgerichts und im Schrifttum anerkannt, dass dieser als Teil des Schutzes der natürlichen Lebensgrundlagen im Rahmen von Art. 20a GG erfasst ist. ${ }^{21}$ Demzufolge teilt Art. 20a GG als Klima-Staatszielbestimmung im Besonderen grundsätzlich die gleichen Rechtswirkungen wie Art. 20a GG als Staatszielbestimmung zum Umweltschutz im Allgemeinen. Das bedeutet im Einzelnen: Art. 20a GG verpflichtet alle Träger der öffentlichen Gewalt auf das Umweltschutzziel. Dabei wirken die Bindungen der Staatszielbestimmung gegenüber den drei Staatsgewalten - Gesetzgebung, Rechtsprechung, vollziehende Gewalt unterschiedlich. ${ }^{22}$ Für den Gesetzgeber wirkt Art. 20a GG als Handlungsauftrag, für die Exekutive bei der Anwendung von einfachgesetzlichen Normen interpretationsund ermessensleitend und für die Judikative im Rahmen der Kontrolle der Verwaltung ebenso leitend bei der Auslegung von Normen und bei der Prüfung von Ermessensund Planungsentscheidungen. ${ }^{23}$

10) BT-Drs. 19/4522, S. 3

11) Vgl. Franzius, Recht und Politik 2019, 230 ff.; Calliess, Der Klimaschutz führt ein verfassungsrechtliches Schattendasein, in: Konrad Adenauer Stiftung, 2019; Maslaton, NJ 2019, $427 \mathrm{ff}$.

12) Cremer, Ausschuss-Drs. 19(4)214 D; Degenhart, AusschussDrs. 19(4)214 A; Gärditz, Ausschuss-Drs. 19(4)214 F; Proelß, Ausschuss-Drs. 19(4)214 B; Saurer, Ausschuss-Drs. 19(4)214 E; Vosgerau, Ausschuss-Drs. 19(4)214 C.

13) Vgl. Kahl, BayVBl. 2009, $97 \mathrm{ff}$.

14) Art. 150 WRV lautet: „Die Denkmäler der Kunst, der Geschichte und der Natur sowie die Landschaft genießen den Schutz und die Pflege des Staates."

15) Kloepfer, DVB1. 1988, 305, 306; Scholz, in: Maunz/Dürig, GG, 89. EL 10/2019, Art. 20a Rdnr. 4.

16) Der Arbeitskreis sozialdemokratischer Juristen schlug folgenden Normtext vor: ,Jeder hat ein Recht auf ein Leben in menschenwürdiger Umwelt. (...)“, s. in ZRP 1972, 76, 77.

17) Bundesminister des Innern/Bundesminister der Justiz (Hrsg.), Bericht der Sachverständigenkommission „Staatszielbestimmungen/Gesetzgebungsaufträge“, 1983, Rdnr. $130 \mathrm{ff}$.

18) Ipsen, Über das Grundgesetz, 1988, S. 8 f.

19) Vgl. Bundesminister des Innern/Bundesminister der Justiz (Hrsg.), Bericht der Sachverständigenkommission „Staatszielbestimmungen/Gesetzgebungsaufträge“", 1983, Rdnr. 7.

20) BGB1. I S. 3146, am 15.11.1994 in Kraft getreten, geändert durch Gesetz v. 26. 7.2002, BGBl. I S. 2826 (Aufnahme des Tierschutzes in Art. 20a GG),

21) Vgl. BVerfG, Urt. v. 13.3.2007 - 1 BvF 1/50, BVerfGE 118, 79, 110; BVerfG, Urt. v. 5.11.2014 - 1 BvF 3/11, 137, 350, 369; BVerwG, Urt. v. 25.1.2006-8 C 13.05, BVerwGE 125, 68, 71; aus dem Schrifttum u.a. Schulze-Fielitz, in: Dreier, GG, Bd. 2, 3. Aufl. 2015, Art. 20a Rdnr. 32; Epiney, in: v. Mangoldt/Klein/ Starck, GG, Bd. 2, 7. Aufl. 2018, Art. 20a Rdnr. 18; Gärditz, in: Landmann/Rohmer, Umweltrecht, 91. EL 9/2019, GG, Art. 20a Rdnr. 9.

22) Hierzu vgl. z. B. Gärditz, in: Landmann/Rohmer, Umweltrecht, 91. EL 9/2019, GG, Art. 20a Rdnr. 27 ff.; Kloepfer, Umweltrecht, 4. Aufl. 2016, $42 \mathrm{ff}$

23) Sommermann, in: von Münch/Kunig, GG, Bd. 1, 6. Aufl. 2012, Art. 20a Rdnr. $37 \mathrm{ff}$.; Schulze-Fielitz, in: Dreier, GG, Bd. 2, 3. Aufl. 2015, Art. 20a GG Rdnr. 67 ff. 
Art. 20a GG erlangt als Klima-Staatszielbestimmung praktische Effektivität, wenn der Gesetzgeber das Ziel und die Mittel hierzu angemessen durch einfaches Recht konkretisiert. ${ }^{24}$ Die Justiziabilität der gesetzgeberischen Entscheidung zur Konkretisierung der Staatszielbestimmung ist allerdings sehr stark begrenzt. Die verfassungsgerichtliche Kontrolle der Wahrung einer Staatszielbestimmung beschränkt sich auf evidente Verstöße ${ }^{25}$. Dies folgt aus dem weiten gesetzgeberischen Gestaltungsspielraum, der im Hinblick auf eine funktionsadäquate Kompetenzverteilung/Gewaltengliederung und einen demokratischen Willensbildungsprozess geboten ist. ${ }^{26}$ Den weiten gesetzgeberischen Gestaltungsspielraum hat das Bundesverfassungsgericht auch in seiner Rechtsprechung zu Art. 20a GG zugrunde gelegt. ${ }^{27}$ Es macht keine konkreten Vorgaben in Bezug auf das materielle Schutzniveu. So wird rechtspraktisch betont, dass das Bundesverfassungsgericht „,nicht Vordenker eines konkreten Umweltschutzprogramms" sein will. ${ }^{28}$ Ein evidenter Verstoß und damit ausnahmsweise justiziabel ist die Verletzung des Zielkerns von Art. 20a GG. ${ }^{29}$ Dies betrifft ein Mindestmaß an Umweltschutz bzw. Klimaschutz zur Gewährung des ,ökologischen Existenzminimums", auf das noch eingegangen wird.

Der Schutzauftrag des Gesetzgebers geht aber über den engen justiziablen Kontrollbereich hinaus. Er umfasst ein Optimierungsgebot ${ }^{30}$, das sich auch auf die Bewältigung des Klimawandels bezieht. Das bedeutet keinen absoluten Vorrang des Klimaschutzes vor anderen Verfassungsgütern. Vielmehr ist nach dem Prinzip der praktischen Konkordanz der Klimaschutz mit anderen Verfassungsprinzipien und Verfassungsgütern in Ausgleich zu bringen. ${ }^{31}$ Das Optimierungsgebot begründet zugleich eine gesetzgeberische Pflicht zur (fortlaufenden) Evaluierung und ggf. Nachbesserung von Gesetzen zur Anpassung an die neuesten - validierten - Erkenntnisse in Wissenschaft und Technik. Auch resultiert daraus eine Pflicht des Gesetzgebers zur Begründung, wie er dem Klima-Staatsziel Rechnung trägt. ${ }^{32}$ Zur Erfüllung der Begründungslast ist das Instrument der Gesetzesfolgenabschätzung geeignet. ${ }^{33}$ Für die organisationsrechtliche Anbindung einer Prüfung der Klimaverträglichkeit von Gesetzen kommen künftig verschiedene Formen in Betracht. Zu erwägen ist die Anbindung an dem bestehenden Normenkontrollrat. ${ }^{34}$ Des Weiteren wäre zu überlegen, welche Rolle der Expertenrat für Klimafragen nach $\$ \$ \$ 11$, 12 Bundes-Klimaschutzgesetz de lege ferenda im Rahmen der Prüfung einnehmen könnte. Von der Schaffung eines eigenen externen Expertengremiums mit einem Vetorecht im Gesetzgebungsverfahren ${ }^{35}$ ist indes vor dem Hintergrund demokratietheoretischer Bedenken abzuraten. ${ }^{36}$

\subsection{Grundrechtsverstärkende und-einschränkende Wirkung}

Eine weitere materiell-rechtliche Dimension erlangt die Klima-Staatszielbestimmung in Verbindung mit Grundrechten. Originär begründet die Staatszielbestimmung objektives Verfassungsrecht, das keine subjektiven Rechte für den Einzelnen, also kein Klimaschutzgrundrecht konstituiert. Allerdings kann die Staatszielbestimmung modifizierend auf den Grundrechtsschutz einwirken - dies betrifft die Konstellation der Grundrechtseingriffe, aber auch die der staatlichen Schutzpflichten. In dem Zusammenhang sind die sich mehrenden Stimmen im Schrifttum zur Anerkennung eines Grundrechts auf ein ökologisches Existenzminimum anzuführen. Dieses leitet sich verfassungsdogmatisch aus der Menschenwürde nach Art. 1 Abs. 1 GG in Verbindung mit der staatlichen Verpflichtung zum Schutz von Leben und Gesundheit in Art. 2 Abs. 2 und der natürlichen Lebensgrundlagen nach Art. 20a GG ab. ${ }^{37}$ Für die Entfaltung von Maßstäben für die substantiierte Darlegung von ökologischen Notfällen könnte möglicherweise das Konzept planetarer Belastungsgrenzen fruchtbar gemacht werden. ${ }^{38}$ Dabei ist zugleich auf die Komplexi- tät und die Schwierigkeit der Ermittlung des Sicherheitsabstandes zu den Belastungsgrenzen hinzuweisen. Die mittelbaren und ungewissen Kausalzusammenhänge zwischen Schädigung des Klimas und einer Gesundheits- oder gar Lebensbeeinträchtigung sind ebenso schwierig nachweisbar. ${ }^{39}$ Auf solche bzw. ähnlich gelagerte Schwierigkeiten deutet auch die im Ergebnis erfolglos gebliebene KlimaKlage vor dem Verwaltungsgericht Berlin (hier im Hinblick auf die Eigentumsgarantie der klagenden Landwirte) hin. ${ }^{40}$ Abzuwarten bleibt, ob und inwieweit das Bundesverfassungsgericht auf die Frage des ökologischen Existenzminimums in den noch laufenden Verfahren zu den KlimaVerfassungsbeschwerden ${ }^{41}$ eingeht.

Andererseits ist Klimaschutz ein hochrangiger Verfassungsbelang, der erhebliche Eingriffe in die Freiheitsgrundrechte der Berufsfreiheit nach Art. 12 Abs. 1 GG, der Eigentumsgarantie nach Art. 14 I und des Grundrechts auf freie Entfaltung der Persönlichkeit nach Art. 2 I GG erlauben kann. Bei Abwägungen kollidierender Interessen nimmt der Klimaschutz besonderes Gewicht ein. ${ }^{42}$

24) Vgl. für Staatszielbestimmungen im Allgemeinen Kahl, Nachhaltigkeitsverfassung, 2018, S. 26; Hahn, Staatszielbestimmungen im integrierten Bundesstaat, 2010, S. 417.

25) Murswiek, in: Sachs, GG, 8. Aufl. 2018, Art. 20a Rdnr. 57.

26) Vgl. Sommermann, in: von Münch/Kunig, GG, Bd. 1, 6. Aufl. 2012, Art. 20a Rdnr. 38.

27) Siehe u.a. BVerfG, Urt. v. 13.3.2007 - 1 BvF 1/50, BVerfGE 118, 79, 110 (Treibhausgas-Emissionsberechtigungen); BVerfG, Urt. v. 24.11.2010 - 1 BvF 2/05, BVerfGE 128, 1 (Gentechnikgesetz); BVerfG, Urt. v. 17.12.2013 - 1 BvR 3139/08, 1 BvR 3386/08, BVerfGE 134, 242, 289 (Garzweiler).

28) Voßkuhle, NVwZ 2013, 1, 4.

29) Sommermann, in: von Münch/Kunig, GG, Bd. 1, 6. Aufl. 2012, Art. 20a Rdnr. 39.

30) Epiney, in: v. Mangoldt/Klein/Starck, GG, Bd. 2, 7. Aufl. 2018 , Art. 20a Rdnr. 62.

31) Kloepfer, Umweltrecht, 4. Aufl. 2016, $\$ 3$ Rdnr. 25; Kahl, Nachhaltigkeitsverfassung, 2018, S. 26.

32) Vgl. Schulze-Fielitz, in: Dreier, GG, Bd. 2, 3. Aufl. 2015, Art. 20a Rdnr. 72f.; Gassner, DVBl. 2013, 547, 550; Kahl, Nachhaltigkeitsverfassung, 2018, S. 27; Wieland, ZUR 2016, 473, 480.

33) Dazu Kahl, in: Kluth/Krings (Hrsg.), Gesetzgebung, 2014, $\$ 13$.

34) Hierfür bedürfte es der Erweiterung des Prüfauftrages des Normenkontrollrates nach $₫ 1$ III Gesetz zur Einsetzung eines Nationalen Normenkontrollrates (NKRG) v. 14.8.2006, BGBl. I S. 1866; zuletzt geändert durch Art. 1 des Gesetzes v. 16.3.2011, BGBl. I S. 420

35) Siehe z. B. den Vorschlag des Sachverständigenrates für Umweltfragen, SRU, Demokratisch regieren in ökologischen Grenzen - Zur Legitimation von Umweltpolitik, Sondergutachten 2019, S. $143 \mathrm{ff}$.

36) Ebenso ablehnend Franzius, Recht und Politik 2019, 230, 237.

37) Prägend zum Begriff „ökologisches Existenzminimum“ Rupp, JZ 1971, 404; Scholz, JuS 1976, 232, 234; ferner zum Ganzen Rauschning, VVDStRL 38, 167, 170 ff., $186 \mathrm{ff}$.; Hoppe, VVDStRL 38, 211, 216 ff.; Kloepfer, Grundrecht auf Umweltschutz, 1978, S. 22, 27; Bock, Umweltschutz im Spiegel von Verfassungsrecht und Verfassungspolitik, 1990, S. 119 ff.; Schulz-Fielitz, in: Dreier, GG, Bd. 2, 3. Aufl. 2015, Art. 20a Rdnr. 43 f.; Murswiek, in: Sachs, GG, 8. Aufl. 2018, Art. 20a Rdnr. 41, Scholz, in: Maunz/ Dürig, GG, 89. EL 10/2019, Art. 20a Rdnr. 8; Gärditz, in: Landmann/Rohmer, Umweltrecht, 91. EL 9/2019, GG, Art. 20a GG Rdnr. 78; Calliess, Rechtsstaat und Umweltstaat, 2001, S. 300; ders., Der Klimaschutz führt ein verfassungsrechtliches Schattendasein, in: Konrad Adenauer Stiftung, 2019, S. 8; siehe auch Frenz, UPR 2020, $1 \mathrm{ff}$; ähnlich Kahl, Nachhaltigkeitsverfassung, 2018, S. 31; kritisch Voßkuhle, NVwZ 2013, 1, 6.

38) Hierzu Calliess, Rechtsstaat und Umweltstaat, 2019, S. 9; ders., ZUR 2019, $385 \mathrm{ff}$.

39) Zu diesem Problemkreis auch Meyer, NJW 2020, 894, $895 \mathrm{ff}$; Groß, NVwZ 2020, 337, $340 \mathrm{f}$.

40) Die Klage wurde wegen mangelnder Klagebefugnis als unzulässig abgewiesen, VG Berlin, Urteil v. 31.10.2019, $10 \mathrm{~K}$ 412.18, juris.

41) Siehe oben Fn. 6.

42) Vgl. Gärditz, in: Landmann/Rohmer, Umweltrecht, 91. EL 9/2019, GG, Art. 20a Rdnr. 60 f. 


\subsection{Verfassungskulturelle und politische Wirkungen}

Durch ein Staatsziel wird zudem die Staatstätigkeit programmatisch ausgerichtet und das öffentliche Bewusstsein identitätsstiftend mitgeprägt. ${ }^{43}$ Der ,Bedarf an symbolischer Vergewisserung über eine gesellschaftliche Bewusstseinsbildung" ${ }^{44}$ kann positiv aufgenommen werden. Eine Staatszielbestimmung - so auch zum Umwelt- und Klimaschutz - kann zu einer Integrationswirkung der Verfassung beitragen. ${ }^{45}$ Mit ihr wird ein Leitbild verfassungsrechtlich verankert, das für den Staat richtungsweisend zur Bewältigung des Menschheitsproblems des Klimawandels ist. Wie Staatszielbestimmungen im Allgemeinen kann die Klima-Staatszielbestimmung im Besonderen neben rechtlichen Wirkungen auch politische und verfassungskulturelle Signal- sowie Appellwirkungen entfalten. ${ }^{46}$

\section{Landesverfassungsrecht}

\subsection{Föderaler Mehrwert eines Staatsziels im Landesverfassungsrecht}

Im Hinblick auf die bereits erfasste Schutzwirkung des Klimas im Rahmen der Staatszielbestimmung des Art. 20a GG auch gegenüber den drei Staatsgewalten der Bundesländer erhebt sich die Frage, ob eine eigene landesverfassungsrechtliche Staatszielbestimmung einen Mehrwert aufweist. Die Beantwortung dieser Frage hängt zunächst vom Grundverständnis des deutschen Föderalismus ab. Dieser geht mit der eigenen Staatlichkeit der Bundesländer davon aus, dass in den Ländern eigene Vollverfassungen existieren können. Selbst wenn eine Verfassungsnorm im Grundgesetz bereits verankert ist, kann es politischen und rechtlichen Sinn machen, diese auch in der Landesverfassung aufzunehmen. Vom Selbstverständnis einer vollen Landesverfassung ausgehend würde auch die Verankerung einer Parallelnorm zu Art. 20a GG mit der zusätzlichen expliziten Aufnahme des Klimaschutzes einen verfassungsrechtlichen föderalen Ertrag herbeiführen. Darüber hinaus ist es durchaus möglich, dass die Landesverfassungsgerichtsbarkeit die Staatszielbestimmung der Landesverfassung anders auslegt als die Parallelnorm im Grundgesetz. ${ }^{47}$ Auch liegt es an dem Landesgesetzgeber, in welchem Umfang und welcher Intensität er der Klima-Staatszielbestimmung im Rahmen seiner Kompetenzen rechtspraktische Wirksamkeit verleiht. Der Landesgesetzgeber kann einen eigenen landesspezifischen Klima-Weg gehen. Die Aufnahme einer neuen expliziten Staatszielbestimmung zum Klimaschutz kann als Ausdruck eines gelebten Föderalismus mit seiner freiheitssichernden und demokratiestärkenden Funktion und als Raum für Innovationen verstanden werden. ${ }^{48}$

\subsection{Geltende Umwelt-Staatsziele im landesföderalen Vergleich}

Die Geschichte des föderalen Umweltstaates der Bundesrepublik Deutschland zeigt, dass sich auch im Umweltrecht grundlegende Vorzüge des Föderalismus realisieren. Dies gilt insbesondere für die Innovationskraft föderaler Strukturen. ${ }^{49}$ So sind nicht nur verschiedene Umweltfachgesetze zuerst auf Landesebene (wie auch die Klimaschutzgesetze) ${ }^{50}$ und dann erst auf Bundesebene entstanden. Auch im Hinblick auf die Verankerung des Staatsziels Umwelt in die Verfassung waren die Länder Vorreiter gegenüber dem Bund (1994). Als erstes Bundesland hat Baden-Württemberg bereits 1976 die Staatszielbestimmung Umweltschutz in die Landesverfassung (Art. 86) aufgenommen. Es folgten 1984 Bayern (Art. 3 Abs. 2, s. auch Art. 141 Abs. 1), 1985 Saarland (Art. 59a), Nordrhein-Westfalen (Art. 29a) und RheinlandPfalz (zuerst in Art. 73a, nunmehr Art. 6)), 1986 Bremen (Art. 11a) und Hamburg (Präambel), 1990 Berlin (zuerst Art. 21a, nun Art. 31) und Schleswig-Holstein (zuerst in Art. 7, nunmehr in Art. 11), 1991 Hessen (früher Art. 26a, jetzt Art. 26b), 1993 Niedersachsen (Art. 1 Abs. 2), die fünf neuen Bundesländer: 1992 Sachsen (Art. 10), Brandenburg (Art. 39, 40), Sachsen-Anhalt (Art. 35) und 1993 Mecklenburg-Vorpommern (Art. 12) und Thüringen (Art. 31). Eine Gesamtschau der landesverfassungsrechtlichen Staatszielbestimmungen ergibt, dass diese unterschiedlich gefasst sind. Einige sind ähnlich wie Art. 20a GG kurz gefasst, einige aber auch ausdifferenzierter. Auch wenn bei den ausdifferenzierten Staatszielbestimmungen im Ergebnis das materielle Schutzniveau substantiell bislang wohl kaum über das von Art. 20a GG hinausgeht, werden mit ihnen landesspezifische Prioritäten deutlich. Exemplarisch hierfür ist die Brandenburgische Verfassung hervorzuheben, die gleich an mehreren Stellen den Umweltschutz anführt - in der Präambel, in Art. 2 Abs. 1 als Staatsstrukturprinzip, in Art. 28 als Bildungsziel, in Art. 31 als Schranke für die Wissenschaftsfreiheit bei der Zerstörung natürlicher Lebensgrundlagen, in Art. 39 als Zentralnorm für den Umweltschutz, in Art. 42 Abs. 2 als Bestandteil der marktwirtschaftlichen Ordnung, in Art. 43 im Hinblick auf die Land- und Forstwirtschaft und in Art. 101 Abs. 1 als ökologische Funktion für den Haushalt. Die Zentralnorm Art. 39 BbgVerf weist im Vergleich zu Art. 20a GG einige Besonderheiten auf. So ist nach Art. 39 Abs. 1 BbgVerf der Umweltschutz auch die Pflicht ,aller Menschen“ und damit als eine Grundpflicht verankert. Eine solche bzw. ähnliche Norm findet sich auch in einigen anderen Landesverfassungen. ${ }^{51}$ Des Weiteren hat nach Art. 39 Abs. 2 BbgVerf jeder das „Recht auf Schutz seiner Unversehrtheit vor Verletzungen und unzumutbaren Gefährdungen, die aus Veränderungen der natürlichen Lebensgrundlagen entstehen“. Hiermit ist zugleich das Grundrecht auf Garantie eines „ökologischen Existenzminimums“ fundiert. ${ }^{52}$ Auf ein allgemeines Umweltgrundrecht scheint der Normtext des Art. 141 Abs. 3 S. 1 BayVerf hinzudeuten (,Der Genuss der Naturschönheiten (...) ist jedermann gestattet"). Der BayVerfGH ${ }^{53}$ hat dies jedoch ausdrücklich verneint. Soweit ersichtlich, hat bislang kein Landesverfassungsgericht eine modifizierte Dogmatik zu einer UmweltStaatszielbestimmung entwickelt, die von der des Bundesverfassungsgerichts zu Art. 20a GG abweichen würde. Die Rechtspraxis zur Heranziehung des Umwelt-Staatsziels bewegt sich in den auch für Art. 20a GG gewohnten Bahnen. Dies trifft ebenfalls für das bekannte Urteil des OVG Berlin-Brandenburg vom 3.11.2011 ${ }^{54} \mathrm{zu}$ dem Heizpilzverbot

43) Vgl. zu Staatszielbestimmungen allgemein BT-Drs. 12/6000, 1993, S. 65, 77; Isensee, in: Isensee/Kirchhof, Handbuch des Staatsrechts, Bd. IV, 3. Aufl. 2006, \$73 Rdnr. 6; Murswiek, in: Sachs, GG, 8. Aufl. 2018, Art. 20a Rdnr. 12-16 zu den Funktionen von Art. 20a GG.

44) Gärditz, Atomausstieg in Grundgesetz?, 2016, S. 64.

45) Vgl. Sternberger, in: ders., Schriften, Bd. X: Verfassungspatriotismus, 1990, S. $3 \mathrm{ff}$.

46) Vgl. Kahl, Nachhaltigkeitsverfassung, 2018, S. 25.

47) Vgl. Kahl, BayVBl. 2009, 97, 105.

48) $\mathrm{Zu}$ den Funktionen des Föderalismus vgl. Härtel, in: dies. (Hrsg.), Handbuch Föderalismus: Föderalismus als demokratische Rechtsordnung und Rechtskultur in Deutschland, Europa und der Welt, Bd. I, 2012, S. $3 \mathrm{ff}$.

49) Kloepfer, in: Härtel (Hrsg.), Handbuch Föderalismus, Bd. III, 2012, \$68 Rdnr. 6. Zu den Vorteilen des Föderalismus im Allgemeinen vgl. u. a. Härtel, in: dies. (Hrsg.), Handbuch Föderalismus, Bd. I, 2012, S. 3 ff.

50) Dies betrifft z. B. auch die Immissionsschutz-, Bodenschutz- und Abfallgesetze.

51) Vgl. Art. 141 Abs. 1, 2 S. 2 BayVerf, Art. 12 Abs. 3 MeVOVerf, Art. 69 Abs. 1 RhPfVerf, Art. 59a SaarVerf, Art. 10 Abs. 1 SaVer; Art. 35 Abs. 2 SaAnVerf, Art. 31 Abs. 1 ThürVerf.

52) Vgl. Lieber/Iwers/Ernst, Praxis der Komunalverwaltung, Art. 39 BbgVerf, S. 289.

53) BayVerfGH, Urt. v. 27.7.2011 - Vf. 5-VII-10, BeckRS 2011, $53258=$, juris Rdnr. 46.

54) OVG Berlin-Brandenburg, Urt. v. 3.11.2011 - OVG 1 B 65/10, BeckRS 9988, 81918, S. 5. 
für die Außengastronomie in Berlin zu. Rekurriert wurde in dieser Rechtssache auf das den Privatinteressen überwiegende öffentliche Interesse am Klimaschutz, das gem. Art. 31 BerlVerf und Art. 20a GG Verfassungsrang hat.

\section{Klima-Staatsziel in Hamburg, aber gescheiterte Entwürfe in Bayern und Schleswig-Holstein}

In Hamburg ist die Einführung des Klima-Staatsziels auf einen gemeinsamen Antrag von SPD, CDU, Grüne und Die Linke zurückzuführen. Am 20.2.2020 ist die Staatszielbestimmung zum Schutz der natürlichen Lebensgrundlagen in der Präambel um den folgenden Satz ergänzt worden: „Insbesondere nimmt die Freie und Hansestadt Hamburg ihre Verantwortung für die Begrenzung der Erderwärmung wahr." ${ }^{55}$ Die Gesetzesbegründung gibt näheren Aufschluss über die intendierte Wirkung des expliziten KlimaStaatsziels. Betont wird die Bedeutung des Klimaschutzes bei Abwägungsentscheidungen auf Landesebene, die weiter zu stärken sei. Die Begrenzung der Erderwärmung wird als eine „,vorrangige staatliche Aufgabe“ qualifiziert. ${ }^{56}$

Auch nach dem in Schleswig-Holstein von der SPDFraktion eingebrachten Gesetzentwurf vom 14.2.2019 sollte der Schutz des Klimas Bestandteil des Staatsziels des Schutzes der natürlichen Lebensgrundlagen werden. Art. 11 Verf Schl-Hol sollte die Formulierung tragen: „Die natürlichen Grundlagen des Lebens, insbesondere das Klima, sowie die Tiere stehen unter dem besonderen Schutz des Landes, der Gemeinden und Gemeindeverbände sowie der anderen Träger der öffentlichen Verwaltung." Mit dieser Regelungsweise und dem Wort ,insbesondere“ besteht eine Ahnlichkeit zu der Klimabestimmung in Hamburg. In der Gesetzesbegründung wird auf die besondere Verantwortung des Landes zum Klimaschutz verwiesen. Auf einen möglichen expliziten Vorrang des Klimaschutzes gegenüber dem Gewässerschutz oder der Biodiversität wird in der Begründung nicht eingegangen. Das Wort „insbesondere“, das üblicherweise bei der Auslegung von Rechtsvorschriften als eine nicht abschließende Aufzählung verstanden wird, könnte hier aber durchaus aufgrund der Semantik der Norm auf eine stärkere Gewichtung des Klimas hindeuten. ${ }^{58}$ Die Landtagsabgeordneten der Jamaika-Koalition (CDU, Grüne, FDP) stimmten am 22.1.2020 gegen den SPD-Antrag, der Südschleswigsche Wählerverband (SSW) enthielt sich. Auch wenn die Grünen in Schleswig-Holstein die Verankerung des ausdrücklichen Klima-Staatsziels inhaltlich befürworten, stimmten sie aus Koalitionstreue gegen den Antrag. ${ }^{59}$

In Bayern sollten nach dem Gesetzentwurf der CSUFraktion und der Fraktion Freie Wähler vom 16.1.2019 die in Art. 141 Abs. 1 S. 4 der Verfassung genannten Schutzgüter um das Schutzgut Klima ergänzt werden. Die Staatszielbestimmung sollte lauten: „Es gehört auch zu den vorrangigen Aufgaben von Staat, Gemeinden und Körperschaften des öffentlichen Rechts Boden, Wasser, Luft und Klima als natürliche Lebensgrundlagen $\mathrm{zu}$ schützen, eingetretene Schäden möglichst zu beheben oder auszugleichen und auf möglichst sparsamen Umgang mit Energie zu achten. "60 In der Gesetzesbegründung ${ }^{61}$ heißt es, dass das Ausmaß der Herausforderung Klimaschutz es erfordert, „die Bedeutung des Klimaschutzes bei solchen Abwägungsentscheidungen klar zu benennen und weiter zu stärken“. Damit ist aber kein Klimavorrang gemeint, wie ihn etwa die Initiative „Bayerisches Energiewende-Volksbegehren Klimaschutz in die Verfassung" einfordert. ${ }^{62}$ Im Februar 2019 scheiterte die Aufnahme des Staatsziels Klimaschutz in die Landesverfassung an der nötigen Zwei-Drittel-Mehrheit im Landtag.

\section{Gesetzentwürfe zum Klima-Staatsziel in Niedersachsen}

Bei allen der drei in Niedersachsen vorgeschlagenen Regelungen der Fraktionen handelt es sich der verfassungsrecht- lichen Konzeption nach um Staatszielbestimmungen. Der Vorschlag von SPD und CDU hat die Verantwortung für die zukünftigen Generationen aufgenommen und bezieht sich auf Klimaschutz sowie Klimawandelanpassung. Auch der FDP-Vorschlag erstreckt sich auf beide Säulen der Bewältigung des Klimawandels - auf Klimaschutz und Klimawandelanpassung. Er ist an das internationale Klimarecht angelehnt und nimmt den bedeutsamen Grundsatz der gemeinsamen, aber unterschiedlichen Verantwortung auf. Sowohl der Vorschlag von SPD und CDU als auch der Vorschlag der FDP enthalten verfassungsmäßiges Verfassungsrecht im Sinne von Art. 46 NdsVerf (Revisionssperrklausel) und Art. 28 Abs. 1 S. 1 GG (Homogenitätsklausel). Der Vorschlag der Fraktion Bündnis 90/Die Grünen geht in seiner Direktionskraft über die beiden anderen Vorschläge hinaus, begegnet aber deutlichen verfassungsrechtlichen Bedenken. Gegenüber dem Grünen-Vorschlag eines Klimavorrangs setzen die Vorschläge von SPD, CDU und FDP beim Klimaschutz auf den Ausgleich mit anderen Verfassungsgütern.

\subsection{Der Gesetzentwurf der Fraktion der SPD} und der Fraktion der CDU

\section{„Artikel 6c}

Klimaschutz und Klimaanpassung

In Verantwortung auch für die künftigen Generationen schützt das Land das Klima und mindert Folgen des Klimawandels."

\subsubsection{Inhalt und Bedeutung des Gesetzentwurfs}

Diese Verfassungsnorm enthält eine Verpflichtung des Landes Niedersachsen zum Klimaschutz und eine Verantwortung auch für künftige Generationen in dieser Hinsicht. Neben dem Klimaschutz (Mitigation) erstreckt sich die Staatszielbestimmung auch auf die Anpassung an den Klimawandel (Adaption). Der Wortlaut ,mindert die Folgen des Klimawandels" deutet auf diese Interpretation hin. Bestätigt wird diese Lesart durch die Überschrift des Artikels „Klimaanpassung“ und die Gesetzesbegründung $(, \ldots$ sich auf die Folgen des Klimawandels einzustellen"). Art. 6c NdsVerf ist als reine Staatszielbestimmung $\mathrm{zu}$ qualifizieren, es werden keine konkreten Pflichten für das Land bestimmt. Wie auch die Gesetzesbegründung ${ }^{63}$ ausführt, soll der Art. 6c als ,objektiv-rechtlicher Verfassungssatz (...) den Staat“ verpflichten und ,richtet sich an den Landesgesetzgeber, an Judikative und Exekutive. Sie ist als Auslegungshilfe oder im Rahmen von Abwägungsentscheidungen heranziehbar." Der Entwurf entspricht einer klassischen offenen Formulierung einer Staatszielbestimmung in einer Verfassung. Er kommt damit zugleich dem

55) Eingefügt durch das 19. Gesetz zur Änderung der Verfassung der Freien und Hansestadt Hamburg vom 20.2.2020, HmbGVBl. 2020 Nr. 10, S. 145; zur Begründung s. Drs. 21/19680 (gemeinsamer Antrag von SPD, CDU, Grüne und Die Linke).

56) Drs. 21/19680, S. 2.

57) Drs. 19/1273.

58) Tobias Koch (CDU-Landtagsabgeordneter in Schleswig-Holstein) hat in seiner Rede vom 22.1.2020 im Landtag die Lesart des Klimavorrangs zugrunde gelegt, Stand 20.7.2020, abrufbar unter https://www.tobias-koch-cdu.de/artikel/nachhaltiger-klimaschutz-gelingt-auch-ohne-verfassungsaenderung.

59) Schleswig-Holsteinischer Landtag, Pressemeldung, Klimaschutz: Verfassung, Verkehr, Moore, Wald, 22.1.2020.

60) Dieser Normtext zeigt, dass auch bereits der geltende Art. 141 Abs. 1 S. 4 BayVerf mit dem Gebot des Energiesparens ein zentrales Klimaschutzinstrument - die Energieeffizienz - inkorporiert hat.

61) LT-Drs. 18/101.

62) Klimaschutz in die Verfassung, Bayerisches EnergiewendeVolksbegehren, Stand 20.7.2020, abrufbar unter https://klimaschutz-in-die-verfassung.de/.

63) LT-Drs 18/4839, S. 9. 
Gebot der politischen Gestaltungsverantwortung des parlamentarischen Gesetzgebers entgegen. Der äußerst hohen Komplexität des Klimaschutzes und der Klimawandelanpassung wird dadurch Rechnung getragen, dass die Entwurfsfassung eine differenzierende Politik mit einer großen Bandbreite an Instrumenten ermöglicht und eine praktische Konkordanz kollidierender Belange herstellen lässt. In diesem Zusammenhang wird auch das umfassendere Prinzip der Nachhaltigkeit mit der Integration des Ökologischen, Ökonomischen und Sozialen in intergenerativer Perspektive miteinbezogen.

Im Hinblick auf die Vereinbarkeit mit der Revisionssperrklausel des Art. 46 Abs. 2 NdsVerf bestehen keine Bedenken. Nach Art. 46 Abs. 2 NdsVerf sind Verfassungsänderungen unzulässig, wenn sie den in Art. 1 Abs. 2 und Art. 2 niedergelegten Grundsätze widersprechen. In Art. 1 Abs. 2 NdsVerf ist auch die Staatszielbestimmung ,Schutz der natürlichen Lebensgrundlagen" verankert. Der Entwurf zum Klima-Ziel würde aber keine Änderung (im Sinne einer Abschaffung oder Reduktion), sondern eine Konkretisierung der Staatszielbestimmung ,Schutz natürlicher Lebensgrundlagen" darstellen. Des Weiteren ist die Homogenitätsklausel des Art. 28 Abs. 1 S. 1 GG gewahrt.

\subsubsection{Mehrwert gegenüber der aktuellen Rechtslage}

Es stellt sich die Frage, ob mit der neuen Staatszielbestimmung überhaupt ein Mehrwert gegenüber der geltenden Verfassungsrechtslage geschaffen wird. Die Umwelt-Staatszielbestimmung in Art. 1 Abs. 2 NdsVerf erfasst wie Art. 20a GG auch den Klimaschutz. Im Gegensatz zu Art. 20a GG fehlt im geltenden Art. 1 Abs. 2 NdsVerf allerdings der Verweis ,,in Verantwortung für die künftigen Generationen“" und damit die explizite Betonung des Nachhaltigkeitsprinzips mit seiner generationenübergreifenden Wirkung. Indem Art. 6c NdsVerf auch die Zukunftsverantwortung des Landes für den Klimaschutz ausdrücklich verankert, konstituiert er für das Landesverfassungsrecht einen Mehrwert. Die Niedersächsische Verfassung wird damit in Bezug auf die Bewältigung des Klimawandels um das ökologische Nachhaltigkeitsprinzip mit seiner intertemporalen Dimension bereichert. Ein weiterer Mehrwert der neuen Verfassungsnorm ist darin zu konstatieren, dass die Klimaanpassung ausdrücklich Verfassungsrang erhält.

\subsection{Der Gesetzentwurf der Fraktion der FDP}

\section{„Artikel 6c}

\section{Klimaschutz, Klimaanpassung}

Das Land Niedersachsen setzt sich in seinem Handeln in Wahrnehmung der gemeinsamen, aber unterschiedlichen Verantwortung innerhalb der internationalen Gemeinschaft dafür ein, die Erderwärmung zu begrenzen und die Menschheit wirksam vor den Folgen des Klimawandels zu schützen."

\subsubsection{Inhalt und Bedeutung des Gesetzentwurfs}

Das Land Niedersachsen wird dazu verpflichtet, sich für die Begrenzung der Erderwärmung (Klimaschutz) und den Schutz der Menschen vor den Folgen des Klimawandels (Klimaanpassung) einzusetzen. Positiv zu bewerten ist die begriffliche Verdeutlichung des Klimaschutzes in seinen anthropogenen Verursachungsformen - es geht konkret um die Begrenzung der Erderwärmung. Klimaschutz und Klimawandelanpassung richten sich expressis verbis auf den Schutz der Menschheit und benennt damit den Schutzadressaten. Sie können sich aber auch auf den Schutz der ökologischen Ressourcen beziehen, da diese wohl zumindest mittelbar auch zu einem Schutz der Menschheit beitragen. Semantisch ist die Gesamtheit der Menschheit angesprochen. Kompetenzrechtlich sind hier allerdings Grenzen gesetzt. Denn die staatliche Verantwortung des Landes Niedersachsen kann sich nur auf die Bereiche der Landeskompetenz beziehen. Der vorgelegte Entwurf ist in seiner Formulierung aber insofern gerechtfertigt, als die Menschen in Niedersachsen als Teil der Gesamtheit der Menschheit begriffen werden können. Dies wird dadurch unterstrichen, dass der Gesetzentwurf der FDPFraktion zur Klima-Staatszielbestimmung den Grundsatz der gemeinsamen, aber unterschiedlichen Verantwortung (,,common but differentiated responsibilities“) aufnimmt. In seiner vollständigen Bezeichnung im Klimavölkerrecht gehört im Übrigen noch der Zusatz ,.... and respective capabilities" (" ...und ihren jeweiligen Fähigkeiten") hinzu.

Dieser Grundsatz stammt aus dem internationalen Klimaschutzrecht (vgl. Art. 3 Nr. 1 und 4 Klimarahmenkonvention sowie 3. Erwägungsgrund der Präambel Übereinkommen von Paris). ${ }^{64}$ Durch diesen Grundsatz wird anerkannt, dass alle Staaten eine Verantwortung in Sachen Klimaschutz haben. Gleichzeitig wird jedoch auch klargestellt, dass die „Verantwortungs-Grade“ der Staaten sich unterscheiden (so insbesondere beim Vergleich von Industrienationen, Schwellenländern, ,,armen“"Ländern etc.). Mit Aufnahme dieses Grundsatzes in die Landesverfassung könnte auch auf diese unterschiedlichen Verantwortungsgrade verwiesen werden. Dies könnte als eine Anerkennung der besonderen Verantwortung Niedersachsens als Teil des föderal-strukturierten Staatswesens des Industriestaates Deutschland zu verstehen sein. Der Grundsatz der gemeinsamen, aber unterschiedlichen Verantwortung ist von hoher Bedeutung und wurde in jüngster Zeit in Klima-Judikaten in Bezug genommen, so z.B. in dem Urteil des Niederländischen Hoge Raad vom 20.12.2019 in der Rs. Urgenda ${ }^{65}$ und in Urteil des VG Berlin vom 31.10.201966. Im Hinblick auf die konkrete Bedeutung des Grundsatzes für Niedersachsen wäre eine Klarstellung in der Gesetzesbegründung aufzunehmen. Eine Aufnahme dieses zentralen Grundsatzes des Klimavölkerrechts in die Landesverfassung ist in Bezug auf den materiellen Prüfungsmaßstab des Art. 46 NdsVerf nicht zu beanstanden. Auch die Homogenitätsklausel des Art. 28 Abs. 1 GG ist gewahrt. Im Vergleich zum Entwurf der SPD-Fraktion/CDU-Fraktion und der Grünen-Fraktion weist der Text des FDP-Entwurfs nicht ausdrücklich auf die für das Land Niedersachsen geltende „Verantwortung für die künftigen Generationen" hin.

\subsubsection{Mehrwert gegenüber der geltenden Rechtslage}

Das Staatsziel Klimaschutz ist zwar bereits implizit von Art. 1 Abs. 2 NdsVerf erfasst. Angesichts der herausragenden Bedeutung und Komplexität der Bewältigung des Klimawandels ist es sinnvoll, Klimaschutz explizit als Staatsziel zu benennen. Außerdem würde neben der Mitigation auch die Adaptation ausdrücklich Verfassungsrang erhalten. Als positiv an dem Entwurf ist zu bewerten, dass die föderale Verantwortung des Landes bei der Bewältigung des globalen Problems des Klimawandels konturiert wird. Künftig wäre zu klären, welche rechtspraktischen Auswirkungen die Aufnahme des Grundsatzes der gemeinsamen, aber unterschiedlichen Verantwortung in die Landesverfassung hätte. Dabei wäre zu fragen, ob hieraus eine qualifizierte Verpflichtung des Landes Niedersachsens als Teil der Bundesrepublik Deutschland zum Klimaschutz erwächst und wie sich dies in konkreten politischen Positionierungen niederschlägt.

\subsection{Der Gesetzentwurf der Fraktion Bündnis 90/Die Grünen}

\section{„Artikel 6c \\ Umweltschutz und Klimaschutz}

64) Zu diesem Grundsatz vgl. u.a. Jahrmarkt, in: Härtel (Hrsg.), Forum Umwelt-, Agrar- und Klimaschutzrecht, Bd. 9, 2016, S. 71 ff., $295 \mathrm{ff}$

65) Dazu ausführlicher unter 6.3

66) VG Berlin, Urt. v. 31.10.2019 - $10 \mathrm{~K}$ 412.18, Rdnr. 82, juris. 
(1) Das Land schützt die Umwelt, die Biodiversität und das Klima auch in Verantwortung für kommende Generationen. (2) Alle Gesetze und Verordnungen werden im Hinblick auf die Auswirkungen auf das Klima und die Einhaltung der durch internationale Abkommen verbindlichen und im Niedersächsischen Klimagesetz festgelegten Klimaziele überprüft. Neue Gesetze und Verordnungen dürfen nur erlassen werden, wenn sie den in völkerrechtlichen Abkommen und im Niedersächsischen Klimagesetz festgelegten verbindlichen Klimazielen nicht widersprechen (Klimavorrang)."

Nach der Gesetzesbegründung sollen hierdurch die ,,auch für das Land Niedersachsen gültige Verpflichtung internationaler Abkommen zum Klimaschutz" hervorgehoben werden. ${ }^{67}$ Weiter heißt es: „Mit dem Klimavorrang werden als Ausfluss aus der Verfassungsänderung und dem neuen Staatsziel Klimaschutz alle Gesetze und Verordnungen im Hinblick auf den Klimaschutz überprüft. Das Prüfergebnis im Hinblick auf den Klimaschutz ist von der Landesregierung bei allen Gesetzen und Verordnungen zu ermitteln und aufzuführen. Gesetze und Verordnungen, die den unter $\$ 4$ [des $\mathrm{Nds}$. Klimagesetzes] erlassenen Klimazielen widersprechen, dürfen nicht erlassen werden, bzw. eine Kompensation in einem anderen Bereich ist durchzuführen." 68

\subsubsection{Staatszielbestimmung Umwelt-, Biodiversitäts- und Klimaschutz (Art. 6c Abs. 1 NdsVerf)}

Die Staatszielbestimmung bezieht sich auf die Umwelt im Allgemeinen, die Biodiversität und das Klima. Die Schutzgüter der natürlichen Lebensgrundlagen, wie sie auch in Art. 1 II NdsVerf verankert sind, werden im Hinblick auf die Biodiversität und das Klima konkretisiert. Eine solche Konkretisierung hat für die Verfassungsinterpretation nur deklaratorische Bedeutung. Wie im Gesetzentwurf der SPD-Fraktion und CDU-Fraktion wird hier ebenfalls die Verantwortung für künftige Generationen aufgenommen. Die damit verbundene Verankerung des Nachhaltigkeitsprinzips ist als ein (föderaler) Mehrwert gegenüber der geltenden Rechtslage anzusehen. Im Vergleich zu den Entwürfen der Fraktionen der SPD und der CDU sowie der FDP bezieht sich die Staatszielbestimmung im Entwurf der Grünen nicht auf die Klimaanpassung. Weder der Wortlaut der Norm oder der Überschrift noch die Gesetzesbegründung lassen hierauf schließen.

5.3.2 Klima-Überprüfungspflicht

(Art. 6c Abs. 2 S. 1 NdsVerf)

Die vorgeschlagene Verfahrensnorm des Art. 6c Abs. 2 S. 1 NdsVerf enthält eine staatliche Pflicht zur Überprüfung von Gesetzen und Verordnungen im Hinblick auf das Klima. Prüfungsmaßstab sind die Auswirkungen auf das Klima, die Einhaltung der durch internationale Abkommen verbindlichen und im Niedersächsischen Klimagesetz festgelegten verbindlichen Klimaziele. Nach dem Wortlaut gilt die Verpflichtung zur Klima-Prüfung nicht nur für künftige Gesetze, sondern für alle Gesetze. Damit sind auch alle bereits erlassenen Landesgesetze umfasst, die weiterhin in Kraft sind. Auch wenn es sich hierbei um eine durchaus konsequente Regelung handelt, dürfte der Aufwand für die Prüfung aller Gesetze erheblich sein. Darüber hinaus ergibt sich aus dem Gesetzentwurf keine Rechtsfolge für den Fall, dass ein Verstoß ,alter“ Gesetze gegen die Klimaschutzziele festgestellt wird (Art. 6c Abs. 2 S. 2 bezieht sich nur auf neue Gesetze und Verordnungen). Auch wenn die Feststellung der Klimaschädlichkeit einzelner Gesetze u. U. zu einem politischen Handlungsdruck führen könnte, würde es vorerst bei einer aufwändigen Prüfung ohne rechtliche Folgen bleiben. Die Begründung des Gesetzentwurfs ${ }^{69}$ sieht vor, dass das Prüfergebnis im Hinblick auf den Klimaschutz von der Landesregierung bei allen Gesetzen und Verordnungen zu ermitteln und aufzuführen ist. Die konkrete In- stitution, die diese umfangreiche Prüfung vorzunehmen hat, wird nicht explizit benannt.

\subsection{3 „Klimavorrang“ (Art. 6c Abs. 2 S. 2 NdsVerf)}

Mit der Einführung eines Klimavorrangs würde eine ganz neue materiell-rechtliche Klima-Verfassungsnorm etabliert werden. Art. 6c Abs. 2 S. 2 NdsVerf enthält dem Wortlaut nach eine absolute materiell-rechtliche Grenze: Wenn neue Gesetze und Verordnungen den in völkerrechtlichen $\mathrm{Ab}$ kommen und im Nds. Klimagesetz ,festgelegten verbindlichen Klimazielen" widersprechen, dürfen sie nicht erlassen werden - m. a. W. verstoßen sie gegen die Niedersächsische Verfassung. Die verbindlichen Klimaziele würden also eine absolute Grenze für die Verfassungsmäßigkeit von Gesetzen darstellen. Sofern ein Widerspruch vorliegt, besteht nach dem Wortlaut des Gesetzentwurfs kein Raum mehr für eine Abwägung mit anderen Belangen. Hierfür sieht der Gesetzentwurf auch die Legaldefinition des „Klimavorrangs" vor. Nach der Gesetzesbegründung soll im Falle eines Widerspruchs zu den Klimazielen durch ein Gesetz bzw. eine Verordnung auch die Möglichkeit zur Kompensation in einem anderen Bereich bestehen. ${ }^{70}$ Der Wortlaut des Art. 6c Abs. 2 S. 2 gibt keinen Anhaltspunkt für eine solche Ausnahme- oder Kompensationsregel her. Wenn eine solche Regel gewollt ist, stellt sich bereits die Frage, wieso sie nicht direkt in den Wortlaut aufgenommen wird. Es besteht also eine Diskrepanz zwischen der vorgeschlagenen Verfassungsnorm und der Gesetzesbegründung. Dieser Gesetzentwurf hätte jedenfalls deutliche Auswirkungen auf die Rechtslage - einerseits aufgrund des verfassungsrechtlichen Verweises auf die internationalen und im Niedersächsischen Klimagesetz vorgesehenen Klimaziele und andererseits aufgrund des sich darauf beziehenden ,Klimavorrangs“. Der Verfassungsrang verbindlicher Klimaziele hätte die verfassungsprozessuale Konsequenz, dass der Staatsgerichtshof etwa im Rahmen einer abstrakten Normenkontrolle gemäß Art. $54 \mathrm{Nr} .3 \mathrm{NdsVerf}$ zu prüfen hätte, ob ein Landesgesetz den jeweiligen verbindlichen Klimazielen widerspricht.

\subsubsection{Verfassungsrang für verbindliche Klimaziele}

Die vorgeschlagene Regelung des Art. 6c Abs. 2 NdsVerf, mit der verbindliche Klimaziele von völkerrechtlichen $\mathrm{Ab}$ kommen und des Niedersächsischen Klimagesetzes Landesverfassungsrang erhalten würden, wirft eine Reihe von Fragen auf.

a) Klimaziele nach dem Niedersächsischen Klimagesetz: Hinsichtlich der Verbindlichkeit der Klimaziele nach dem Nds. Klimagesetz stellen sich zwei Fragen. Zum einen ist die verfassungsrechtlich induzierte Verbindlichkeit der einfachgesetzlichen Klimaziele nicht vollständig kongruent mit dem Wortlaut des $\$ 4 \mathrm{Nds}$. Klimagesetz-Entwurfs. So spricht $\$ 4$ Nds. Klimagesetz davon, dass bestimmte Ziele erreicht werden „sollen“ oder „,angestrebt“ werden. Hier könnte angenommen werden, dass Ziele angestrebt werden, aber ihre Erreichung noch nicht sicher und damit nicht verbindlich ist. Diese Unklarheit hinsichtlich der Verbindlichkeit der niedersächsischen (perfektiblen) Klimaziele müsste behoben werden. Zum anderen würde hier der verfassungsändernde Gesetzgeber die Verweisung als Regelungstechnik wählen, indem die Normen des Nds. Klimagesetzes zu den Klimazielen inkorporiert werden. Mit der Verweisung auf einfaches Recht wird dieses in den Rang des Landesverfassungsrechts gehoben.

Es stellt sich dabei die Frage, ob mit der Verweisung dem Textänderungsgebot gemäß Art. 46 Abs. 1 NdsVerf Rech-

67) LT-Drs. 18/4499, S. 7

68) LT-Drs. $18 / 4499$, S. 8

69) LT-Drs. $18 / 4499$, S. 8.

70) LT-Drs. $18 / 4499$, S. 8 
nung getragen wird. Dieses soll der Rechtsklarheit und Rechtssicherheit dienen. ${ }^{71}$ Im Schrifttum wird die Ansicht vertreten, dass das Textänderungsgebot (nach der Äquivalenznorm Art. 79 Abs. 1 S. 1 GG) nicht nur eine formale Textänderung verlangt, sondern auch, dass die explizite Textänderung dem materiellen Regelungsgehalt der Verfassungsänderung entspricht. ${ }^{72}$ Bei einer dynamischen Verweisung auf andere Rechtsnormen unterhalb des Verfassungsranges würde der geänderte normative Gehalt der Verfassung nicht mehr unmittelbar aus dessen Text ermittelbar sein. ${ }^{73}$ Dem Textänderungsgebot eine solche Reichweite zuzuschreiben, ist indes nach der Rechtsprechung des Bundesverfassungsgerichts nicht zwingend. ${ }^{74}$ Derartige Verweisungen finden sich z. B. in Art. 13 Abs. 3 S. 1, Art. 38 Abs. 2, Art. 28 Abs. 1 S. 3, Art. 44 Abs. 2 S. 1 GG. Verfassungsdogmatisch anders gelagert sind Verweisungen des Grundgesetzes im Sinne eines gesetzgeberischen Konkretisierungsauftrages, wie sich dies z. B. in besonderer Weise im Finanzverfassungsrecht durch das Maßstäbegesetz ${ }^{75}$ ausdrückt; dies genießt jedoch keinen Verfassungsrang.

Bei dynamischen Verweisungen von Verfassungsnormen auf außerverfassungsrechtliche Normen könnten aber allgemeine Bedenken hinsichtlich der Rechtsklarheit bestehen. ${ }^{76}$ Der normative Gehalt des vorgeschlagenen Art. 6c Abs. 2 NdsVerf lässt sich allerdings aufgrund der eingeschränkten Inbezugnahme eines Gesetzes hinsichtlich einer konkreten Regelung - nämlich hinsichtlich der im Nds. Klimagesetz festgelegten Klimaziele - hinreichend klar und bestimmt erfassen; hiervon unberührt ist die oben aufgeworfene Frage der fehlenden vollständigen Kongruenz zur Verbindlichkeit der Klimaziele. Verfassungsrechtliche Bedenken hinsichtlich der dynamischen Verweisung auf unterverfassungsrechtliche Normen (hier einfachgesetzliche Normen) bestehen aber auch vor dem Hintergrund, dass der verfassungsändernde Gesetzgeber einem rangniederen Normautor Einfluss auf den Inhalt der Verfassung einräumt. Es besteht keine eindeutige Linie darüber, welche Regelungen dem verfassungsändernden Gesetzgeber vorbehalten sind und dementsprechend der verfassungsändernde Zugriff des rangniederen Normautors versperrt bleiben muss. ${ }^{77}$ Zwar ist nach dem vorliegenden Regelungskonzept der Landtag ein- und derselbe Gesetzgeber für verfassungsändernde Gesetze und das in Bezug genommene einfache (Klima)Gesetz. Damit bestehen grundsätzlich in der organischen Normenurheberschaft keine Rangunterschiede. Allerdings existieren Unterschiede in den Verfahrensanforderungen (Quoren) und in der Rechtsquellenhierarchie. Aus dem Grunde bleiben die grundsätzlichen Bedenken gegenüber der dynamischen Verweisung prüfungsbedürftig. Auf der Seite des verfassungsmäßigen Verfassungsrechts steht die Verweisungsnorm des Art. 6c Abs. 2 NdsVerf jedenfalls dann, wenn sie verfassungskonform als statische Verweisung ausgelegt wird. Angesichts des offenen Wortlauts der Regelung ist dies möglich.

b) Klimaziele nach dem Völkerrecht: Die Konzeption des Art. 6c Abs. 2 NdsVerf zur Etablierung des Landesverfassungsranges von in völkerrechtlichen Abkommen festgelegten verbindlichen Klimazielen bedarf der Klärung verschiedener verfassungsdogmatischer und verfassungspolitischer Aspekte. Die Verbindlichkeit von Klimazielen, die in völkerrechtlichen Abkommen festgelegt werden, entsteht im deutschen Recht mit der Ratifikation nach Maßgabe des Art. 59 Abs. 2 S. 1 GG. Das hierzu ergangene Transformationsgesetz hat den Charakter eines einfachen Bundesgesetzes. Den Rang zwischen Verfassungsrecht und einfachem Gesetzesrecht besitzen gemäß Art. 25 GG die „,allgemeinen Regeln“" des Völkerrechts. Völkervertragsrecht gehört indes nicht zu den allgemeinen Regeln im Sinne des Art. 25 GG. ${ }^{78}$ Für dieses gilt Art. 59 Abs. 2 GG als lex specialis zu Art. 25 GG und verdrängt diesen. Mit der vorgeschlagenen Regelung in Art. 6c NdsVerf würde entgegen dem Bundesverfassungsrecht eine ,eigene Kategorie von Völkerrecht mit Verfassungsrang" ${ }^{779}$ entstehen. Es stellt sich deshalb die Frage, ob eine solche Landesverfassungsnorm im Verhältnis zum Bundesverfassungsrecht wirksam wäre. Als Ausprägung des Bundesstaates besitzen der Gesamtstaat Staatsqualität und die Gliedstaaten Staatsqualität. Das bedeutet, dass Bund und Länder jeweils ihre eigene, von ihnen selbst bestimmte Verfassung besitzen. ${ }^{80}$ Allerdings wird die Gestaltungsfreiheit der Länder in Bezug auf den Landesverfassungsraum durch das Grundgesetz als gesamtstaatliche Verfassung inhaltlich markiert. ${ }^{81}$ $\mathrm{Ob}$ und inwiefern für das Landesverfassungsrecht eine Bindung an die grundgesetzliche Kompetenzverteilung (insbesondere Art. 70 ff. GG) besteht ${ }^{82}$, bedarf an dieser Stelle keiner abschließenden Erörterung. Denn hier intendiert der verfassungsändernde Gesetzgeber mit Art. 6c Abs. 2 NdsVerf keine Abweichung von den Gesetzgebungskompetenzen des Bundes, wie etwa von der ausschließlichen Zuständigkeit nach Art. 73 Abs. 1 I Nr. 1 GG (,,auswärtige Angelegenheiten“.) Vielmehr würde eine solche neue Landesverfassungsnorm einem ratifizierten völkerrechtlichen Abkommen, das Klimaziele festlegt, anders in die Normenhierarchie einordnen, als es das Grundgesetz konstatiert. Auf die völkerrechtlichen Abkommen zum Klimaschutz, insbesondere für das Übereinkommen von $\mathrm{Pa}$ ris, ist Art. 59 Abs. 2 S. 1 GG anzuwenden. Denn soweit die Abkommen in die Zuständigkeit der Mitgliedstaaten der EU fallen, beziehen sie sich auf Gegenstände der Bundesgesetzgebung. Aus der Anwendung des Art. 59Abs. 2 S. 1 GG folgt deutlich, dass das Transformationsgesetz den Rang eines einfachen Bundesgesetzes in der Normenhierarchie einnimmt. Dieser ausdrückliche bundesverfassungsrechtliche Normbefehl würde durch das Landesverfassungsrecht missachtet werden. Eine solche Kollision von Bundesverfassungsrecht und Landesverfassungsrecht wäre nach der Vorrangregel Art. 31 GG zu lösen: „Bundesrecht bricht Landesrecht". Die vorgeschlagene landesverfassungsrechtliche Regelung zum Verfassungsrang völkerrechtlicher Klimaziele wäre daher unwirksam.

5.3.5 Würdigung des „Klimavorrangs“ im Hinblick auf Schutzgüterkonflikte

Der in Art. 6c Abs. 2 S. 2 NdsVerf vorgeschlagene Klimavorrang wäre zwar ein Instrument im Sinne eines ef-

71) Vgl. Bryde, in: von Münch/Kunig, GG II, 6. Aufl. 2012, Art. 79 Rdnr. 14

72) Vgl. Degenhart, Ausschuss-Drs. 19(4)214 A, S. 5.

73) Vgl. Degenhart, Ausschuss-Drs. 19(4)214 A, S. 5.

74) Vgl. zur Rechtsprechung des BVerfG zur Zulässigkeit dynamischer Verweisungen BVerfG, Urt. v. 15.7.1969 - 2 BvF 1/64, BVerfGE 26, 338, 366f:; BVerfG, Beschl. v. 1.3.1978 - 1 BvR 786/70, 1 BvR 793/70, 1 BvR 168/71, 1 BvR 95/73, BVerfGE, 47, 285, 312 ff.; BVerfG, Urt. v. 23.3.1982 - 2 BvL 13/79, BVerfGE 60, 135, 155

75) Vgl. Rechtsprechung BVerfG, Urt. v. 11.11.1999 - 2 BvF 2/98, $2 \mathrm{BvF} 3 / 98,2 \mathrm{BvF} 1 / 99,2 \mathrm{BvF} 2 / 99$, BVerfGE 101,158.

76) Vgl. Sachs, in: Sachs, GG, 8. Aufl. 2018, Art. 79 Rdnr. 16 i. V.m. Art. 20 Rdnr. 123a.

77) Vgl. Hain, in: v. Mangoldt/Klein/Starck, GG, Bd. 2, 7. Aufl. 2018, Art. 79 Rdnr. 9.

78) BVerfG, Beschl. v. 25.3.1999 - 2 BvE 5/99, BVerfGE 100, 266 , 269; BVerfG, Beschl. 6.12.2006 - 2 BvM 9/03, BVerfGE 117, 141, 149; Cremer, in: Isensee/Kirchhof, Handbuch des Staatsrechts, Bd. XI, 3. Aufl., 2013, \$235 Rdnr. 10; Streinz, in: Sachs, GG, 8. Aufl. 2018, Art. 25 Rdnr. 29.

79) Vgl. Saurer, Ausschuss-Drs. 19(4)214 E, S. 5

80) BVerfG, Beschl. v. 29.1.1974 - 2 BvN 1/69, BVerfGE 36, 342 Rdnr. 45.

81) Vgl. Isensee, SächsVBl. 1994, 30

82) Hierzu überzeugend Huber, in: Sachs, GG, 8. Aufl. 2018, Art. 31 Rdnr. 16f.; a.A.z.B. März, in: v. Mangoldt/Klein/Starck, GG, Bd. 2, 7. Aufl. 2018, Art. 31 Rdnr. 87. 
fektiven Klimaschutzes mit Verfassungsrang. Allerdings bestehen Zweifel daran, ob eine ändernde Regelung zu einem solchen absoluten Klimavorrang, die u.a. eine Verhältnismäßigkeitsprüfung staatlicher Grundrechtseingriffe entfallen lässt, mit der Revisionssperrklausel des Art. 46 Abs. 2 NdsVerf sowie mit dem Homogenitätsgebot des Art. 28 Abs. 1 S. 1 i. V.m. den geschützten Grundsätzen nach Art. 79 Abs. 3 GG $^{83}$ vereinbar wäre. Nach dem Wortlaut des Art. 6c Abs. 2 S. 2 NdsVerf wird eine ,absolute Grenze“, ohne jegliche Ausnahme oder auch eine Möglichkeit des Härtefallausgleichs, festgelegt. Nur in der Gesetzesbegründung wird alternativ zum Nichterlass des Gesetzes eine „Kompensation in einem anderen Bereich“ erwähnt; in dem Fall würde sich der absolute Klimavorrang in einem relativen Klimavorrang gestalten, der eine Güterabwägung ermöglicht und damit nicht den gleichen Bedenken ausgesetzt ist wie ein absoluter Klimavorrang. Nach dem strikten Wortlaut führt Art. 6c Abs. 2 S. 2 NdsVerf jedoch dazu, dass bei einem Widerspruch zu den jeweiligen Klimazielen der Gesetzgeber grundsätzlich keine Abwägung mit anderen Belangen vornehmen kann. Sobald der Widerspruch festgestellt ist, wobei auch diese Beurteilung aus rein tatsächlichen Gründen in bestimmten Fällen sehr komplex sein wird, kann die Regelung in dieser Form nicht mehr ergehen. Die Verhältnismäßigkeitsprüfung und die Auflösung einer Güterkollision im Wege der praktischen Konkordanz entfallen hier. In dieser Perspektive stellt die vorgeschlagene Regelung eine erhebliche Verkürzung des Rechtsstaatsprinzips in seiner Ausprägung als Prinzip der Verhältnismäßigkeit (hier wiederum beim Element der Verhältnismäßigkeit im engeren Sinne) dar. In seiner Grundsatzbedeutung kann auch der Grundsatz der Verhältnismäßigkeit als nicht explizit benanntes Einzelelement des Rechtsstaatsprinzips unberührbar im Sinne des Art. 79 Abs. 3 GG $^{84}$ bzw. der landesverfassungsrechtlichen Revisionsklausel (wie hier nach Art. 46 Abs. 2 NdsVerf) sein.

Das Bundesverfassungsgericht hat indes ausgeführt, dass Art. 79 Abs. 3 GG als Ausnahmevorschrift nicht dazu führen dürfe, dass ,der Gesetzgeber gehindert wird, durch verfassungsänderndes Gesetz auch elementare Verfassungsgrundsätze systemimmanent zu modifizieren“. ${ }^{85}$ Mit der verfassungsändernden Regelung wird das VerhältnismäBigkeitsprinzip zwar nicht in der Gänze preisgegeben. So würden die Elemente der Geeignetheit und Erforderlichkeit auch weiterhin - wenn auch durch die klimaspezifische Vorrangprüfung erheblich modifiziert - bestehen bleiben. Berührt wäre aber das ebenso grundlegende Element der Angemessenheit im Rahmen der VerhältnismäBigkeit bzw. die Verhältnismäßigkeit im engeren Sinne. Auch wenn diese erhebliche Einschränkung der Verhältnismäßigkeitsprüfung nicht allgemein für jeden $\mathrm{Be}-$ reich, sondern speziell für den Bereich des Klimaschutzes erfolgen soll, ist die Reichweite der vorgeschlagenen Norm aufgrund des Querschnittsbezugs des Klimaschutzes in sehr vielen Lebens- und Wirtschaftsfeldern immens. Neben der Qualifizierung dieser absoluten Vorrangregel als verfassungswidrigem Verfassungsrecht wäre die Regelung auch verfassungspolitisch im Lichte des Leitbildes des wohlgeordneten Rechts im Sinne einer guten Klimaschutzgesetzgebung kritisch zu beurteilen. Diese Regelung kann in bestimmten Fällen zur Handlungsunfähigkeit des Gesetzgebers führen, sofern nämlich alle Handlungsmöglichkeiten den Klimaschutzzielen widersprechen. Dies wird aber in bestimmten Szenarien sicher nicht gewollt sein, z.B. dann nicht, wenn ein Gesetz aus sozialen Gesichtspunkten dringend notwendig oder als rechtssichere Grundlage für gemeinwohlorientierte Innovationen geboten ist. Wiederum erlangt der Klimavorrang ausnahmsweise seine verfassungsrechtliche Berechtigung - auch de constitutione lata - für die Konstellation des ,ökologischen Existenzminimums (s. bereits unter 2.2).
Mit einer einseitigen Priorisierung des Klimaschutzes als Schutzgut werden innerhalb des Geltungsbereichs des Klimavorrangs andere ökologische Schutzgüter der natürlichen Lebensgrundlagen zurückgedrängt. Entstehen durch klimaschützende Maßnahmen ökologische Güterkonflikte, würde stets der Klimaschutz Vorrang haben. Die im Bereich des Klimaschutzes auftretenden Umweltzielkonflikte (wie z.B. mit dem Natur-, Gewässer-, Boden- oder Tierschutz) würden keine Abwägung zulassen bzw. keine Herstellung einer praktischen Konkordanz zwischen verschiedenen Belangen verlangen. Der Schutz des Klimas hat immer - und ohne Rücksicht auf den Einzelfall - Vorrang. Dies kann gerade aufgrund der Mehrdimensionalität von Umwelt- und Klimaschutzmaßnahmen nicht gewollt sein. Aus den vorgenannten Gründen stößt ein absoluter Klimavorrang auf erhebliche verfassungsrechtliche Bedenken.

\section{Ausländisches Verfassungsrecht}

Weltweit haben mittlerweile 150 Staaten in ihren Verfassungen Rechte und Bestimmungen über die Umwelt verankert. ${ }^{86}$ Dabei stellt sich die Frage, ob das jeweilige Verfassungsrecht zum Umweltschutz auch den Klimaschutz umfasst, wie das im Verfassungsrecht der EU und in den meisten EU-Mitgliedstaaten der Fall ist. Außerhalb der EU verweisen ausdrücklich auf den Klimaschutz z.B. die Verfassungen von der Dominikanischen Republik ${ }^{87}$, Venezuela ${ }^{88}$ Ecuador $^{89}$, Vietnam ${ }^{90}$, Tunesien ${ }^{91}$, Cote d'Ivoire ${ }^{92}$, Thailand ${ }^{93}$

83) Zur Inbezugnahme der geschützten Grundsätze nach Art. 79 Abs. 3 GG über Art. 28 Abs. 1 S. 1 GG siehe z. B. Nierhaus/Engels, in: Sachs, GG, 8. Aufl. 2018, Art. 28 Rdnr. 9.

84) Vgl. Sachs, in: Sachs, GG, 8. Aufl. 2018, Art. 79 Rdnr. 78.

85) BVerfG, Urt. v. 15.12.1970 - 2 BvF 1/69, 2 BvR 629/68, 2 BvR 308/69, BVerfGE 30,1, $19 \mathrm{f}$.

86) United Nations Environmental Programme (UNEP), Environmental Rule of Law, First Global Report, 2019, S. 2, $156 \mathrm{ff}$.

87) Art. 194 der Verfassung der Dominikanischen Republik (1998/2015): „Die Ausarbeitung und Ausführung eines Plans der territorialen Ordnung durch Gesetz, der die effiziente und nachhaltige Nutzung der natürlichen Ressourcen der Nation gemäß der Notwendigkeit der Anpassung an den Klimawandel gewährleistet, ist eine Priorität des Staates."

88) Art. 127 der Verfassung von Venezuela (1999/2009): „Es ist eine grundlegende Pflicht des Staates, unter aktiver Mitwirkung der Gesellschaft dafür zu sorgen, dass sich die Bevölkerung in einer unbelasteten Umwelt entwickelt, in der Luft, Wasser, Boden, Küsten, Klima, Ozonschicht und lebende Arten nach den gesetzlichen Vorschriften besonderen Schutz genießen.“

89) Art. 414 der Verfassung von Ecuador (2008): „Der Staat ergreift angemessene und sektorübergreifende Maßnahmen zur Eindämmung des Klimawandels, indem er Treibhausgasemissionen, die Entwaldung und die Luftverschmutzung begrenzt; er ergreift Maßnahmen zur Erhaltung des Waldes und der Vegetation und schützt die gefährdete Bevölkerung."

90) Art. 127 der vietnamesischen Verfassung (2013): „Es ist eine grundlegende Pflicht des Staates, unter aktiver Mitwirkung der Gesellschaft dafür zu sorgen, dass sich die Bevölkerung in einer unbelasteten Umwelt entwickelt, in der Luft, Wasser, Boden, Küsten, Klima, Ozonschicht und lebende Arten nach den gesetzlichen Vorschriften besonderen Schutz genießen.“

91) Art. 45 der tunesischen Verfassung (2014) lautet: „Der Staat garantiert das Recht auf eine gesunde und ökologisch ausgewogene Umwelt sowie das Recht auf Mitwirkung am Klimaschutz"

92) Laut Präambel der Verfassung von Cote d'Ivoire (2016) ist die Regierung verpflichtet, zum Klimaschutz beizutragen.

93) Section 258 der thailändischen Verfassung (2017) bezieht sich auf den Schutz der durch den Klimawandel bedrohten Wasserressourcen: „Es werden nationale Reformen in verschiedenen Bereichen durchgeführt, um zumindest Folgendes zu erreichen: (...) ein Wasserressourcen-Managementsystem, das effizient, fair und nachhaltig, unter gebührender Berücksichtigung aller Dimensionen des Wasserbedarfs in Kombination mit Umwelt- und Klimawandel.“ 
und Kuba ${ }^{94}$. In der Schweiz besteht aufgrund einer Volksinitiative die Aussicht auf eine Verankerung eines KlimaArtikels in die Verfassung. ${ }^{95}$ In der EU haben bereits einige Bundesländer in Österreich explizit Klimaschutz in die Landesverfassung aufgenommen. In Frankreich ist die verfassungsrechtliche Verankerung des Klimaschutzes vorgesehen. In den meisten anderen Mitgliedstaaten ${ }^{96}$ lässt sich der Klimaschutz verfassungsrechtlich implizit aus den Staatszielbestimmungen herleiten ${ }^{97}$ und in dreizehn Mitgliedstaaten sogar zusätzlich aus dem Grundrecht auf Umweltschutz ${ }^{98}$ und in einigen Staaten auch aus der Grundpflicht für jedermann zum Umweltschutz ${ }^{99}$. Dies ist eine beachtliche verfassungsrechtliche Entwicklung, auch wenn $\mathrm{zu}$ bedenken ist, dass die Verfassungen in den jeweiligen Staaten unterschiedliche rechtliche Bedeutung und faktische Wirkungskraft besitzen.

\section{1 Österreich}

Auf föderaler Landesebene haben in Österreich bereits die Bundesländer Niederösterreich (2007) ${ }^{100}$, Vorarlberg $(2008)^{101}$ und Tirol (2019) ${ }^{102}$ Klimaschutz als Staatsziel verankert. Ob Klimaschutz als explizites Staatsziel auch in die Bundesverfassung aufgenommen wird, bleibt abzuwarten. Angestrebt wird dies von einem in Gang gesetzten Klimavolksbegehren (Unterschriftensammlung seit 27.8.2019). Die ÖVP warb im Wahlkampf um die Aufnahme des Klimaschutzes in die Verfassung und um die Etablierung eines „Klima-Check“ für neue Gesetze. Der ÖVP wurde entgegengehalten, dass der Klimaschutz bereits als Bestandteil des, umfassenden Umweltschutzes' ${ }^{103}$ in der Verfassung verankert sei. Die neue Koalition von ÖVP und Grüne hat in ihrem Regierungsprogramm angekündigt, dass ein zeitgemäßer verfassungsrechtlicher Kompetenzrahmen zur Erreichung der Klimaziele geprüft und erarbeitet werden soll. ${ }^{104}$

Die Rechtsprechung des österreichischen Bundesverwaltungsgerichts zur Erweiterung des Wiener Flughafens aus dem Jahr $2017^{105}$ verdeutlicht das Potential der Rechtswirkung eines Klima-Staatsziels. Das Gericht entschied, dass die Umwelt- und Klimabelange überwiegten und versagte die Genehmigung. Bei der Anwendung des Fachrechts ${ }^{106}$ zog es $\iint 1$ bis 3 des Bundesverfassungsgesetzes über die Nachhaltigkeit sowie Landesverfassungen heran und stellte fest, dass insbesondere der Klimaschutz als besonders hervorgehobenes Ziel zu berücksichtigen sei. Diese Zielsetzung richte sich nicht nur an den Gesetzgeber, sondern sei auch bei Auslegungs- und Abwägungsfragen zu berücksichtigen. Aufgrund der Gesamtumstände überwiege das Interesse daran, weitere Treibhausgasemissionen zu verhindern. ${ }^{107}$ Der österreichische Verfassungsgerichtshof hob dieses Judikat auf. Er gelangte zu dem Ergebnis, dass bei der Auslegung des einfachen Rechts und der anzustellenden Abwägung gerade nicht auf den Klimaschutz Bezug genommen werden dürfe. Auch könne bei den zu berücksichtigenden Emissionen in Bezug auf deren Feststellung und deren Auswirkungen nur auf das österreichische Staatsgebiet abgestellt werden. ${ }^{108}$ Unter Maßgabe des Urteils des Verfassungsgerichts war der Fall erneut vom Bundesverwaltungsgericht $\mathrm{zu}$ entscheiden. ${ }^{109}$ Auf einen Vorrang der Klimabelange durfte nicht mehr abgestellt werden. Unter Erweiterung von Auflagen wurde der Bau der zusätzlichen Start- und Landebahn schließlich genehmigt. Das Judikat des Verfassungsgerichts ist im juristischen Schrifttum auf unterschiedliche Resonanz gestoßen. ${ }^{110}$

\subsection{Frankreich}

In Frankreich soll Klimaschutz in der Verfassung verankert werden. Die Abgeordneten der französischen Nationalversammlung stimmten am 13.7.2018 für folgende Ergänzung des ersten Verfassungsartikels zu den Grundprinzipien: Frankreich ,handelt (,agir") für den Schutz der Umwelt und der Biodiversität und gegen die Klimaveränderungen“" ${ }^{111}$ Der Conseil d'État hat in seiner Stel- lungnahme vom 20.6.2019 Bedenken gegenüber diesem Normtext vorgebracht. ${ }^{112}$ Diese Formulierung führe $\mathrm{zu}$ nationalen und internationalen Handlungspflichten Frankreichs. Die rechtlichen Konsequenzen hiervon seien unvorhersehbar, insbesondere, wenn der Staat untätig bliebe.

94) Art. 16 lit. f. der kubanischen Verfassung (2019), der in Zusammenhang mit den internationalen Beziehungen steht, bestimmt, dass die Republik Kuba ,den Schutz und die Erhaltung der Umwelt und die Bekämpfung des Klimawandels, der das Überleben der menschlichen Spezies bedroht, auf der Grundlage der Anerkennung gemeinsamer, aber unterschiedlicher Verantwortlichkeiten" fördert.

95) Dazu unter 6.4.

96) Hierzu erhellend Groß, EurUP 2019, 353, 355 f., Umweltverfassungsrechtliche Normen fehlen in Dänemark, Irland und Zypern.

97) Zum Staatsziel Umweltschutz: Art. 7bis Verf Belgien (nachhaltige Entwicklung), Art. 15 Verf Bulgarien, Art. 6 Umweltcharta Frankreich, Art. 24 Abs. 1 Verf Griechenland, Art. 9 Verf Italien (Schutz der Landschaft), Art. 3 Verf Kroatien, Art. 54 Verf Litauen, Art. 11bis Verf Luxemburg, Art. 9 Abs. 2 Verf Malta, Art. 21 Verf Niederlande, $\$ 3$ Öster. Bundesverfassungsgesetz über die Nachhaltigkeit, den Tierschutz, den umfassenden Umweltschutz, die Sicherstellung der Wasser- und Lebensmittelversorgung und die Forschung, Art. 5, 74 Verf Polen, Art. 9 lit. e), 66 Verf Portugal, Art. 135 Abs. 2 lit. e) Verf Rumänien, Kap. $1 \rrbracket 2$ Abs. 3 Verf Schweden, Art. 44 Abs. 4 Verf Slowakei, Art. 72 Abs. 2 Verf Slowenien, Art. 45 Abs. 2 Spanien, Art. XX Abs. 2 Verf Ungarn.

98) Art. 23 Abs. 2 Nr. 4 Verf Belgien, Art. 55 Verf Bulgarien, $\$ 20$ Verf Finnland, Art. 1 Umweltcharta Frankreich, Art. 24 I Verf Griechenland, Art. 115 Verf Lettland, Art. 66 Abs. 1 Verf Portugal, Art. 35 Abs. 1 Verf Rumänien, Art. 44 Abs. 1 Slowakei, Art. 72 Abs. 1 Slowenien, Art. 45 Abs. 1 Verf Spanien, Art. 35 Abs. 1 Grundrechtecharta Tschechien, Art. XXI Abs. 1 Verf Ungarn.

99) $\$ 53$ Verf Estland, Art. 2 Umweltcharta Frankreich, Art. 53 Abs. 3 Verf Litauen, Art. 86 Verf Polen, Art. 66 Abs. 1 Verf Portugal, Art. 44 Abs. 2 Verf Slowakei, Art. 45 Abs. 1 Verf Spanien.

100) Art. 4 Nr. 3 NÖ Landesverfassung: „Das Land Niederösterreich hat in seinem Wirkungsbereich dafür zu sorgen, dass die Lebensbedingungen der niederösterreichischen Bevölkerung in den einzelnen Gemeinden und Regionen des Landes unter Berücksichtigung der abschätzbaren, wirtschaftlichen, sozialen und kulturellen Bedürfnisse gewährleistet sind. Dabei kommen (...) dem Klimaschutz, dem Schutz und der Pflege von Umwelt, Natur, Landschaft und Ortsbild, besondere Bedeutung zu."

101) Art. 7 Abs. 7 Verfassungsgesetz über die Verfassung des Landes Vorarlberg: „Das Land bekennt sich zum Klimaschutz. Zu diesem Zweck fördert das Land Maßnahmen zur Steigerung der Energieeffizienz sowie die nachhaltige Nutzung erneuerbarer Energien, den Betrieb von Atomanlagen hingegen lehnt es ab.“

102) Nach Art. 7 Abs. 3 Tiroler Landesverfassung bekennt sich das Land Tirol ,zu einem nachhaltigen und effektiven Klimaschutz als eine Voraussetzung zum Erhalt unseres Lebensraumes für künftige Generationen".

103) Vgl. $\$ 3$ Abs. 2 Bundesverfassungsgesetz über die Nachhaltigkeit, den Tierschutz, den umfassenden Umweltschutz, die Sicherstellung der Wasser- und Lebensmittelversorgung und die Forschung.

104) Regierungsprogramm 2020-2024, S. 11.

105) BVwG, Erkenntnis v. 2.2.2017, AZ.: W109 2000179-1.

106) ,sonstige öffentliche Belange“" gemäß $\$ 71$ Abs. 1c Luftfahrtgesetz.

107) BVwG, Erkenntnis v. 2.2.2017, AZ.: W109 2000179-1, Ziff. 4.5.14

108) Verfassungsgerichtshof, Erkenntnis v. 29.6.2017, E 875/2017, Rdnr. 201, 204, $223 \mathrm{f}$.

109) BVwG, Erkenntnis v. 23.3.2018, AZ.: W109 2000197-1, Ziff. 4.4.2.

110) Folgend Schmelz, ZVG 2017, $288 \mathrm{fff}$; kritisch Balthasar, ZÖR 2017, 577 ff.; Madner/Schulev-Steindl, ZÖR 2017, 589 ff.

111) Amendement n 328: „Elle agit pour la préservation de l'environnement et de la diversité biologique et contre les changements climatiques". $\mathrm{Zu}$ anderen Formulierungsvorschlägen s. Roux, Zur Aufnahme des Klimaschutzes in die französische Verfassung, in: Würzburger Berichte zum Umweltenergierecht Nr. 38 v. 25.2 .2019 , S. $18-20$.

112) Stand 20.7.2020, abrufbar unter http://www.assemblee-nationale.fr/15/pdf/projets/pl2203-ace.pdf. 
Vorzugswürdig sei nach seiner Ansicht die Formulierung Frankreich ,fördert (,favoriser") die Bewahrung der Umwelt, die Biodiversität und den Klimaschutz." 113 Diesem Vorschlag ist die Regierung in ihrer Vorlage an das Parlament vom 29.8.2019 114 gefolgt. Wie in der verfassungsrechtlichen Diskussion in Deutschland stellt sich die Frage, worin der Mehrwert der Verankerung des Klimaschutzes im Vergleich zur geltenden Rechtslage liegt. Zwar ist bislang der Klimaschutz nicht explizit genannt, aber vom Umweltschutz erfasst. Allerdings könnte durch die Verankerung ein größeres $M a ß$ an Rechtssicherheit und Rechtsklarheit herbeigeführt werden. ${ }^{115}$ Der konkrete materielle Maßstab, der sich aus der neuen Klima-Verfassungsnorm ergibt, bleibt aber schließlich durch die Rechtswissenschaften und bei praktischen Rechtsstreitigkeiten durch den Conseil Constitutionnel (das französische Verfassungsgericht) zu erhellen. ${ }^{116} \mathrm{Im}$ Rahmen einer anhängigen Klima-Klage gegen den Staat vor einem französischen Verwaltungsgericht ${ }^{117}$ werden das geltende französische Umweltverfassungsrecht ${ }^{118}$ und die EMRK ${ }^{119}$ bemüht.

\subsection{Niederlande - Rechtssache Urgenda}

Die höchstrichterliche Rechtsprechung in den Niederlanden zur Verurteilung des Staates zu weitergehenden Klimaschutzmaßnahmen hat internationale Aufmerksamkeit auf sich gezogen. Die Stiftung Urgenda und 886 weitere Privatkläger erhoben 2013 eine sog. Gemeinwohlklage. Anknüpfungspunkt für den Rechtsstreit war die Herabsetzung des Ziels zur Reduktion von Treibhausgasemissionen in den Niederlanden. Bis 2011 verfolgten die Niederlande das Ziel, die Treibhausgasemissionen bis 2020 um 30 Prozent gegenüber $1990 \mathrm{zu}$ reduzieren. Aber nachdem die EU das allgemeine Reduktionsziel auf 20 Prozent festgelegt hatte, wurde das Ziel in den Niederlanden auch auf 20 Prozent herabgesetzt. In Anlehnung an das internationale Klimaschutzrecht beantragte Urgenda beim Bezirksgericht Den Haag, den Staat anzuweisen, den Ausstoß von Treibhausgasen so $\mathrm{zu}$ reduzieren, dass diese Emissionen bis Ende $2020 \mathrm{um}$ 40 Prozent, aber mindestens um 25 Prozent gegenüber 1990 reduziert werden. Erstinstanzlich verurteilte das Bezirksgericht Den Haag in $2015^{120}$ den niederländischen Staat, die Treibhausgase bis Ende 2020 um mindestens 25 Prozent gegenüber $1990 \mathrm{zu}$ reduzieren. Damit ist der niederländische Staat auch zu weitergehenden Klimaschutzmaßnahmen verpflichtet worden. Welche Maßnahmen der Staat hierzu ergreift, steht in seinem Ermessen. Gestützt wurde die Entscheidung auf das (Staats)Haftungsrecht ${ }^{121}$, das im Lichte des Staatsziels zum Umweltschutz nach Art. 21 der niederländischen Verfassung, des Kyoto-Protokolls und den Schutzpflichten aus Art. 2 EMRK (Recht auf Leben) und Art. 8 EMRK (Recht auf Privatheit und Familienschutz) ausgelegt wurde. In zweiter Instanz folgte der Gerichtshof Den Haag mit Urteil vom 9. 10.2018 dem Bezirksgericht im Ergebnis. Allerdings zog es nicht mehr das Haftungsrecht heran, sondern begründete die Pflicht des niederländischen Staates direkt mit Art. 2 und 8 EMRK. ${ }^{122}$ Nach Art. 93 und Art. 94 der niederländischen Verfassung müssen die Gerichte in den Niederlanden die EMRK unmittelbar anwenden. In letzter Instanz bestätigte der Oberste Gerichtshof (Hoge Raad) diese Judikatur mit Urteil vom 20.12.2019.

Im Hinblick auf die Thematik der Klima-Staatszielbestimmung sind vor dem Hintergrund des FDP-Entwurfs in Niedersachsen (s. unter 5.2) die Ausführungen des Hoge Raad zu dem ,Grundsatz der gemeinsamen, aber unterschiedlichen Verantwortung“ von Interesse: Nach Ansicht des Hoge Raad sind die Niederlande (nach Art. 2 und Art. 8 EMRK) verpflichtet, ,,ihren Teil“" zur Verhinderung des gefährlichen Klimawandels beizutragen, auch wenn es bei dem Klimawandel um ein globales Problem handelt. Das Gericht verweist auf Art. 3 Abs. 1 der UN-Klimarahmenkonvention, nach dem die Vertragsparteien das Klimasystem im Ein- klang mit ihren gemeinsamen, aber unterschiedlichen Verantwortlichkeiten und ihren jeweiligen Fähigkeiten schützen müssen. ${ }^{123}$ Zudem betont das Gericht hinsichtlich der Teilverantwortung, dass jedes Land für seinen Teil der Treibhausgasemissionen verantwortlich ist und daher zur Verantwortung gezogen werden kann. Neben dieser Verantwortung für die jeweiligen Emissionen müssen aber auch die Möglichkeiten der jeweiligen Länder zur Reduzierung ihrer Emissionen berücksichtigt werden. ${ }^{124}$ Die von der Regierung vorgebrachten Einwände gegen die staatliche Teilverantwortung lässt das Gericht aufgrund der drohenden schwerwiegenden Folgen des Klimawandels nicht gelten: So sei das Argument, dass ein Staat seine Verantwortung deswegen nicht übernehmen müsse, da andere Länder dies ebenfalls nicht täten, nicht akzeptabel. Genauso steht es um die Argumentation, dass die eigenen Emissionen im globalen Verhältnis so gering seien, dass deren Verringerung global gesehen überhaupt keinen Unterschied mache. ${ }^{125}$ Unbeschadet dessen ist allerdings auf das Argument der Klimawissenschaften hinzuweisen, dass Treibhausgasemissionen keine „nationalstaatliche Fahne“ besitzen und die Bewältigung des Klimawandels dort am effektivsten ist, wo die meisten Emissionen anfallen. Von rechtspraktischem Interesse wird es überdies sein, ob und inwieweit im Rahmen der anhängigen Klima-Klagen die anderen Gerichte Argumente des niederländischen Hoge Raad aufnehmen werden. ${ }^{126}$

113) Stand 20.7.2020, abrufbar unter http://www.assemblee-nationale.fr/15/pdf/projets/pl2203-ace.pdf.

114) Regierungsvorlage Nr. 2203 v. 29.8.2019, Stand 20.7.2020, abrufbar unter http://www.assemblee-nationale.fr/15/pdf/projets/pl2203.pdf, S. 16.

115) http://blog.leclubdesjuristes.com/inscrire-le-climat-a-larticle-1er-de-la-constitution-quelles-consequences/.

116) Vgl. Roux, Zur Aufnahme des Klimaschutzes in die französische Verfassung, in: Würzburger Berichte zum Umweltenergierecht Nr. 38 v. 25.2.2019, S. 22.

117) Zur juristischen Argumentation s. L'Affaire du Siècle, Brief juridique sur la requete deposée au Tribunal Administratif de $\mathrm{Pa}$ ris le 14 Mars 2019, Stand 20.7.2020, abrufbar unter https:// laffairedusiecle.net/wp-content/uploads/2019/03/ADS-Briefjuridique-140319.pdf, S. $9 \mathrm{ff}$.

118) Insbesondere das Recht auf ein Leben in gesundheitsfreundlichen und ausgeglichenen Umweltbedingungen (Art. $1 \mathrm{Um}-$ welt-Charta/Charte de l'environnenment) und die JedermannPflicht zum Umweltschutz (Art. 2 Umwelt-Charta).

119) Recht auf Leben (Art. 2 EMRK) und Recht auf Privatheit und Familie (Art. 8 EMRK).

120) Rechtbank Den Haag, Urt. v. 24.6.2015 - C-09/456689, HA ZA 13-1396, ECLI:NL:RBDHA:2015:7145, englischsprachige Übersetzung, Stand 20.7.2020, abrufbar unter http:// uitspraken.rechtspraak.nl/inziendocument?id=ECLI:NL:RB DHA:2015:7196.

121) Art. 6:162 Zivilgesetzbuch.

122) Gerechtshof Den Haag, Urt. v. 9.10.2018, 200.178.245/01, ECLI:NL:GHDHA:2018:2610, englischsprachige Übersetzung, Stand 20.7.2020, abrufbar unter https://uitspraken.rechtspraak.nl/inziendocument?id=ECLI:NL:GHDHA:2018:2610.

123) HogeRaad,Urt.v.20.12.2019,19/00135,ECLI:NL:HR:2019:2007, Anm. 5.7.1; englischsprachige Übersetzung, Stand 20.7.2020, abrufbar unter https://uitspraken.rechtspraak.nl/inziendocume nt?id=ECLI:NL:HR:2019:2007.

124) HogeRaad,Urt.v.20.12.2019,19/00135,ECLI:NL:HR:2019:2007, Anm. 5.7.6.

125) HogeRaad,Urt.v.20.12.2019,19/00135,ECLI:NL:HR:2019:2007, Anm. 5.7.7.

126) Positiv aufnehmend für die mögliche Rechtsprechung u. a. Gro $\beta$, NVwZ 2020, 337, 338 ff., kritisch Wegener, Stand 20.7.2020, abrufbar unter verfassungsblog.de/urgenda-iii-die-niederlandeals-modell-richterlichen-klimaschutzes/; s. bereits ansatzweise VG Berlin, Urt. v. 31.10.2019 - 10 K 412.18, Rdnr. 84, EnWZ 2020, 88; anmerkend hierzu auch Kahl, EnWZ 2020, 93 f; Meyer, NJW 2020, $894 \mathrm{ff.;}$ im Hinblick auf das zweitinstanzliche Urgenda-Urteil v. 9.10.2018 und die Frage der Übertragbarkeit auf das deutsche Recht s. Voland, NVwZ 2019, 114, $116 \mathrm{ff}$. 


\subsection{Schweiz}

In der Schweiz sollte bereits nach der im Jahre 2008 bei der Bundeskanzlei eingereichten Volksinitiative ,für ein gesundes Klima“" ein Klima-Artikel in die Bundesverfassung eingeführt worden. ${ }^{127}$ Diese Initiative wurde aber in 2012 unter der Maßgabe des Erlasses eines neuen Bundesgesetzes über die Reduktion von $\mathrm{CO}_{2}$ zurückgezogen. ${ }^{128}$ Der Entwurf des $\mathrm{CO}_{2}-$ Gesetzes wurde als indirekter Gegenvorschlag der Initiative durch den Bundesrat forciert. Am 19.12.2019 ist eine neue eidgenössische Volksinitiative „Für ein gesundes Klima (Gletscher-Initiative)“ bei der Bundeskanzlei eingereicht worden. Danach soll in der Bundesverfassung als verbindliches Klimaziel verankert werden, dass in der Schweiz (ähnlich wie in der EU nach dem Green Deal) die Treibhausgasemissionen bis spätestens 2050 auf null reduziert werden. ${ }^{129}$ Zugleich soll geregelt werden, dass die Klimapolitik in der Schweiz auf eine Stärkung der Volkswirtschaft und auf Sozialverträglichkeit ausgerichtet wird und auch Instrumente der Innovations- und Technologieförderung nutzt. Der Bundesrat hat am 3.4.2020 die Erarbeitung eines direkten Gegenentwurfs hierzu in Auftrag gegeben. ${ }^{130} \mathrm{Er}$ hat sich auch für die Aufnahme des Netto-Null-Ziels in die Verfassung positioniert, da hierdurch frühzeitig Planungsund Investitionssicherheit für Wirtschaft und Private herbeigeführt werde. ${ }^{131}$ Im Gegensatz zur Gletscher-Initiative solle aber kein explizites Verbot fossiler Energieträger in der Verfassung aufgenommen werden. Nach Ansicht des Bundesrates sollte der Gestaltungsspielraum des Parlaments und der Kantone bei der Umsetzung des Netto-Null-Ziels erhalten bleiben. Neben der anvisierten Verfassungsänderung ist die anhängige Klimaklage der Vereins KlimaSeniorinnen und von vier weiteren Frauen gegen das eidgenössische Umweltdepartment anzuführen. ${ }^{132}$

\section{Resümee}

Der deutsche Föderalismus erweist sich im Klimaschutzrecht wie im sonstigen Umweltrecht als Motor und Innovationsraum. Dies gilt für das einfache Recht und für das Verfassungsrecht. Auf Landesebene gab es zuerst Umweltfachgesetze und auch ausdrückliche Klimaschutzgesetze. Erst im Anschluss daran zog die Bundesgesetzgebung nach. Auch die verfassungsrechtliche Verankerung des Umweltschutzes erfolgte zuerst in den Landesverfassungen, bevor sie auf Bundesebene in Art. 20a GG ihren Niederschlag fand. Den gleichen Entwicklungspfad könnte es im Hinblick auf eine Klima-Staatszielbestimmung geben. Hamburg als erstes Bundesland mit einer Klima-Staatszielbestimmung in der Verfassung könnte hier der Vorreiter sein. Bei einer Aufnahme einer Klima-Staatszielbestimmung in die Verfassung kommen verschiedene Normierungsoptionen in Betracht, wie auch die Hamburger Bestimmung und vorgestellten Entwürfe in Niedersachsen, Schleswig-Holstein und Bayern belegen. Dabei würde aber eine Regelung zu einem (absoluten) Klimavorrang, wie ihn z.B. der Entwurf der Grünen in Niedersachsen vorsieht, verfassungswidriges Verfassungsrecht darstellen. Geboten bleibt auch bei einer Klima-Verfassungsnorm eine Verhältnismäßigkeitsprüfung staatlicher Grundrechtseingriffe und die Auflösung einer Güterkollision im Wege der praktischen Konkordanz. Eine stärkere Gewichtung des Klimaschutzes im Rahmen staatlicher Entscheidungen, wie dies die Hamburger Regelung vorzeichnet, ist indes ein verfassungsmäßiger Weg. Diese Norm könnte zugleich zu einer dogmatischen Weiterentwicklung der Verhältnismäßigkeitsprüfung im Rahmen von Klimarechtsakten anstoßen. Während im
Grundgesetz eine Klima-Zielbestimmung de constitutione ferenda auf völkerrechtliche Ziele Bezug nehmen dürfte ${ }^{133}$, ist dies in der Landesverfassung aufgrund des Art. 59 Abs. 2 S. 1, Art. 25 GG nicht weiterführend; eine landesverfassungsrechtliche Regelung zum Verfassungsrang völkerrechtlicher Ziele (wie im Nds. Grünen-Entwurf) wäre nach der Vorrangregel Art. 31 GG unwirksam. Aus verfassungstheoretischer Sicht bliebe gegenüber einer expliziten Konstitutionalisierung einer Klima-Staatszielbestimmung noch der Einwand, dass Klimaschutz bereits von Art. 20a GG sowie den Umwelt-Staatszielbestimmungen der Landesverfassungen erfasst und deshalb eine Verfassungsänderung hier nicht erforderlich ist. In der Tat ebnet bereits das gegenwärtige Verfassungsrecht den Weg für ein effektives Klimaschutzrecht. Allerdings kann eine Staatszielbestimmung auch zu einer Integrationswirkung der Verfassung beitragen. Die verfassungskulturelle und politische Wirkung einer Klima-Staatszielbestimmung ist in dieser Perspektive als ein gewichtiges Argument zu bewerten. Dies gilt gleichermaßen für andere Staaten, in denen die Aufnahme einer Klima-Staatszielbestimmung in die Verfassung voraussichtlich bevorsteht, so z.B. in Frankreich und in der Schweiz.

Open Access. Dieser Artikel wird unter der Creative Commons Namensnennung 4.0 International Lizenz veröffentlicht, welche die Nutzung, Vervielfältigung, Bearbeitung, Verbreitung und Wiedergabe in jeglichem Medium und Format erlaubt, sofern Sie den/die ursprünglichen Autor(en) und die Quelle ordnungsgemäß nennen, einen Link zur Creative Commons Lizenz beifügen und angeben, $o b$ Änderungen vorgenommen wurden.

Die in diesem Artikel enthaltenen Bilder und sonstiges Drittmaterial unterliegen ebenfalls der genannten Creative Commons Lizenz, sofern sich aus der Abbildungslegende nichts anderes ergibt. Sofern das betreffende Material nicht unter der genannten Creative Commons Lizenz steht und die betreffende Handlung nicht nach gesetzlichen Vorschriften erlaubt ist, ist für die oben aufgeführten Weiterverwendungen des Materials die Einwilligung des jeweiligen Rechteinhabers einzuholen.

Weitere Details zur Lizenz entnehmen Sie bitte der Lizenzinformation auf http://creativecommons.org/licenses/by/4.0/deed.de.

Open access funding provided by Projekt DEAL.

127) Art. 89a der Bundesverfassung, BBl. 2008, 2579f., Stand 5. 4.2020 abrufbar unter https://www.bk.admin.ch/ch/d/pore/ vi/vis354.html. Hierzu bereits Kahl, BayVBl. 2009, 97, 99.

128) BB1. 2012, 5640.

129) Der Vorschlag eines „Art. 74a Klimapolitik“, Stand 5.4.2020, abrufbar unter https://www.bk.admin.ch/ch/d/pore/vi/vis498. html. Es sollen nicht mehr Treibhausgase ausgestoßen werden dürfen, als natürliche und technische Speicher aufnehmen können.

130) Beauftragt worden hiermit ist das Department für Umwelt, Verkehr, Energie und Kommunikation (Uvek).

131) Stand 20.7.2020, abrufbar unter https://www.admin.ch/gov/ $\mathrm{de} /$ start/dokumentation/medienmitteilungen.msg-id-78667. html.

132) Das Bundesverwaltungsgericht wies die Klage mit Urt. v. 27.11.2018 - A-2992/2017, als unzulässig zurück, Stand 20.7.2020, abrufbar unter https://www.bvger.ch/bvger/de/ home/medien/medienmitteilungen-archiv-2002-2016/ 2018/klimaseniorinnen-unterliegen-vor-gericht.html. Dagegen wurde beim Bundesgericht Beschwerde erhoben. Nach Ansicht der Klägerinnen verletze die Schweiz insbesondere ihre Schutzpflichten aus Art. 2 und Art. 8 EMRK, indem sie nicht die notwendigen Maßnahmen zur Verringerung der Treibhausgasemissionen treffe.

133) Siehe Cremer, ZUR 2019, 278, $280 \mathrm{ff}$. 